\title{
Detailed Derivations of Formulas for Heat Release Rate Calculations Revisited: A Pedagogical and Systematic Approach
}

\begin{abstract}
Jiann C. Yang
National Institute of Standards and Technology, Gaithersburg, MD 20899 USA

jiann.yang@nist.gov

The derivations of the formulas for heat release rate calculations are revisited based on the oxygen consumption principle. A systematic, structured, and pedagogical approach to formulate the problem and derive the generalized formulas with fewer assumptions is used. The operation of oxygen consumption calorimetry is treated as a chemical flow process, the problem is formulated in matrix notation, and the associated material balances using the tie component concept commonly used in chemical engineering practices are solved. The derivation procedure described is intuitive and easy to follow. Inclusion of other chemical species in the measurements and calculations can be easily implemented using the generalized framework developed here.
\end{abstract}

Key words: fire measurement; heat release rate; oxygen consumption calorimetry.

Accepted: May 7, 2019

Published: June 24, 2019

https://doi.org/10.6028/jres.124.016

\section{Glossary}

$\begin{array}{lll}\frac{\text { Symbol }}{A_{\mathrm{L}}} & & \frac{\text { Units }}{\mathrm{m}^{2}} \\ E & & \mathrm{~J} / \mathrm{kg} \\ k_{1}, k_{2} & & \\ M & & \mathrm{~kg} / \mathrm{mol} \\ n & \mathrm{~mol} / \mathrm{s} \\ \hat{n}_{\mathrm{T}, \mathrm{L}} & \mathrm{mol} / \mathrm{s} \\ p & \mathrm{~Pa} \\ p_{\text {vap }} & \mathrm{Pa} \\ q & \mathrm{~W} \\ R H & \\ S & \\ T & \mathrm{~K} \\ v_{\mathrm{L}} & \mathrm{m} / \mathrm{s} \\ y & \mathrm{~mol} / \mathrm{mol} \\ \alpha & \end{array}$

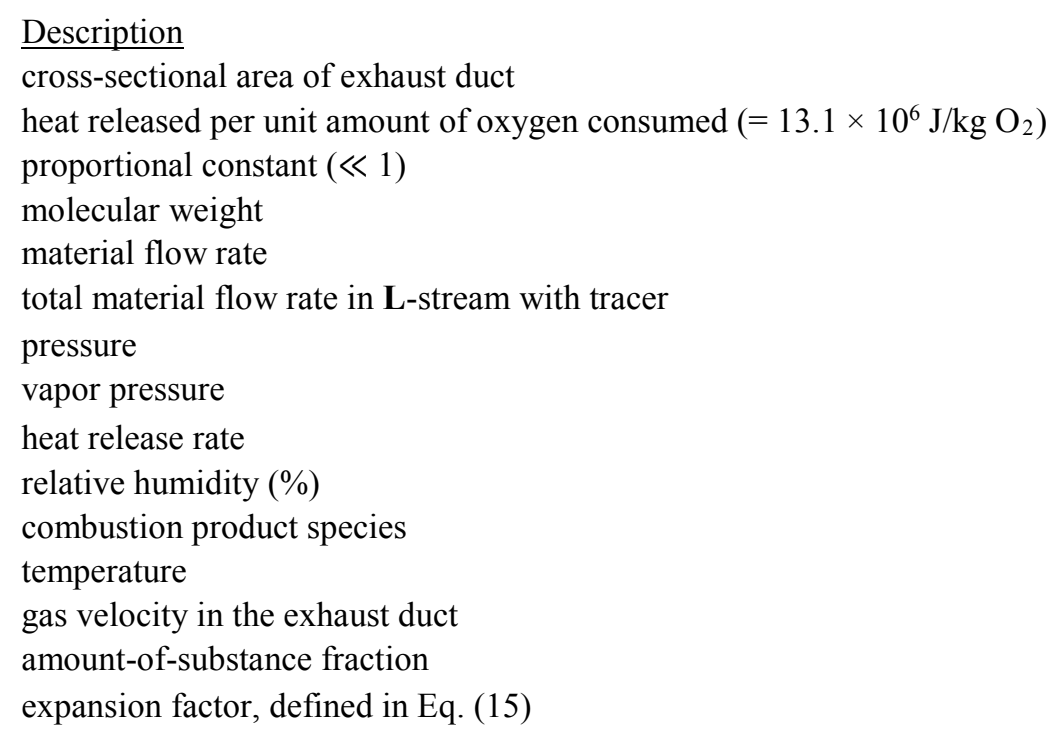




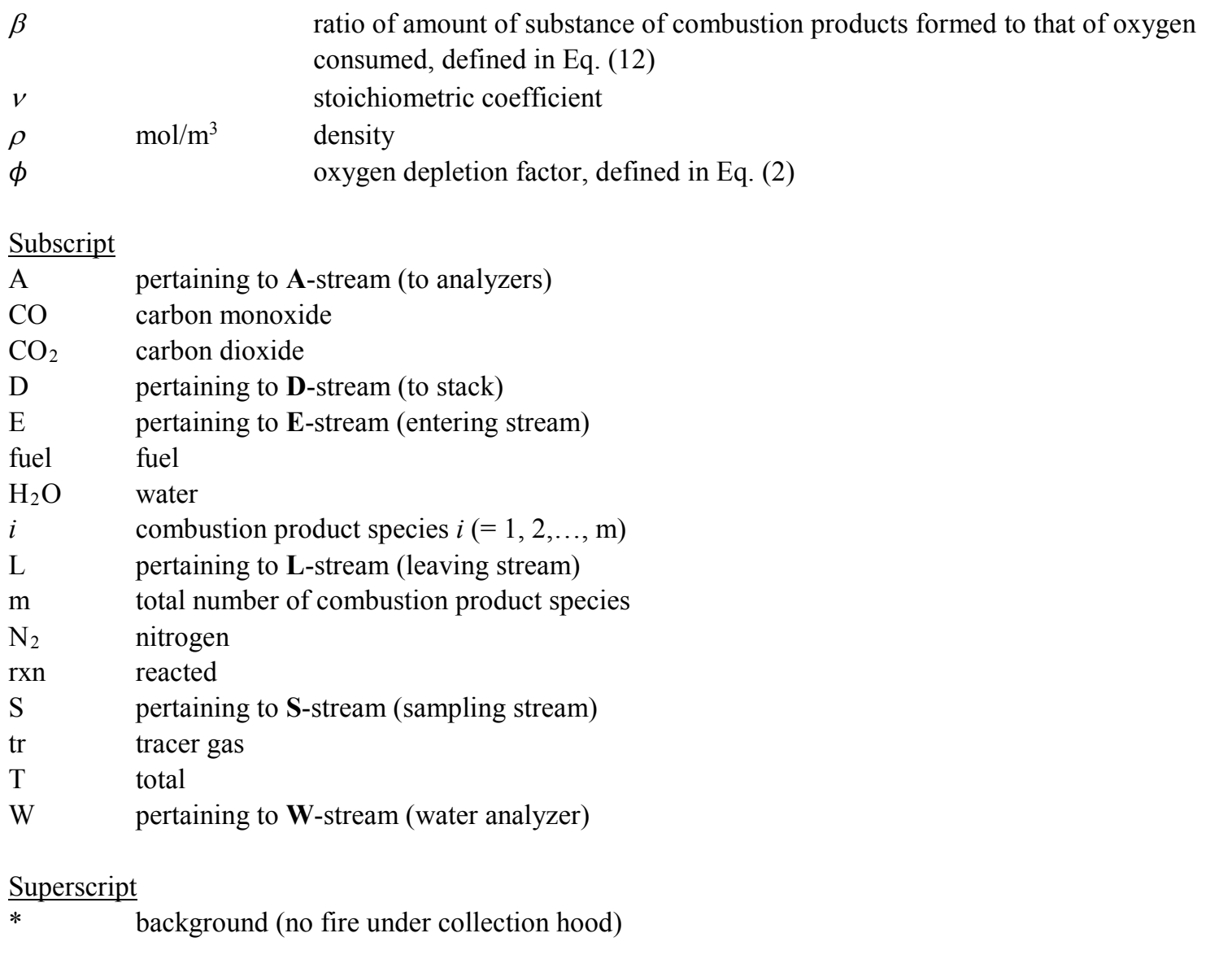

\section{Introduction}

Heat release rate, defined as the amount of energy released by a burning material per unit time, is considered to be the single most important parameter required to characterize the intensity and size of a fire and other fire hazards [1]. The determination of heat release rate can be straightforward if the effective heat of combustion and the burning rate of the tested material are both known. Then, the heat release rate is simply the product of the effective heat of combustion and the burning rate. However, the effective heats of combustion for most materials used in fire tests are generally not known, and measuring burning rates of materials in large-scale fire tests proves quite challenging. Other means need to be developed to measure heat release rates. One such technique is oxygen consumption calorimetry.

In 1917, Thornton [2] observed that the heat released per unit amount of oxygen consumed during the complete combustion of a large number of organic gases and liquids was relatively constant. His finding subsequently formed the basis for modern heat release measurements based on the oxygen consumption principle, from bench-scale cone calorimeters [3] to full-scale fire tests [4]. In 1980, Huggett, at the thenNBS (National Bureau of Standards), expanded Thornton's work by including typical fuels commonly encountered in fires in his study and recommended an average value of $13.1 \mathrm{MJ}$ of heat released per kilogram of $\mathrm{O}_{2}$ consumed for all fuels with $\mathrm{a} \pm 5 \%$ variation [5]. In 1982, Parker published his seminal work on a detailed derivation of a set of formulas that could be used for heat release calculations using the oxygen consumption principle in an NBS publication [6] and later published a different version of the derivation in Ref. [7]. Janssens [8] revisited the derivations using mass basis instead of the volumetric basis 
used by Parker [6, 7]. Janssens and Parker also summarized the formulas in a chapter of a handbook [9]. Brohez et al. [10] modified the heat release rate formulas for sooty fires. More recently, Chow and Han [11] also presented formulas including soot as an additional species.

The derivations of the formulas in the literature $[8,10,11]$ followed essentially the same procedure developed by Parker [6, 7], with little variance. Although the derivations carried out by Parker [6,7] and others $[8,10,11]$ were comprehensive, the approach did not appear to be structured and systematic, and the line of reasoning sometimes was not intuitive. Filling in some of the missing intermediate steps in the derivations was not effortless and required some thinking and assumed knowledge. The use of cumbersome nomenclature, and a myriad of subscripts, superscripts, and super-superscripts, also burdened the readers. Without a complete appreciation for the subtleties involved in the derivation of these equations and the underlying assumptions, incorrect use of the equations or typographical errors introduced inadvertently during transcription of the equations from the literature sources would hardly be noticed by the users, as evidenced by the survey conducted by Lattimer and Beitel [12] on the standard test methods that used the oxygen consumption principle to calculate heat release rates. Of the 17 domestic, foreign, and international standards they reviewed and examined, 12 were identified to have various typographical errors, resulting in 22 incorrect equations in all, and the misprints were found to propagate from standard to standard, most likely attributed to cut-and-paste processes and the poor notations used.

It is necessary to seek a more systematic treatment than those previously presented in the literature. This work is intended to provide a clear and detailed description of the derivations of the formulas used in heat release rate calculations and to make the discussion as general as possible in a consistent and unified manner without introducing additional complexity. The approach presented here is different in many respects from the literature. First, a structured, step-by-step, and pedagogical approach was adopted. For completeness and clarity, this unavoidably led to some of the derivation procedure appearing repetitious in the following discussion. Second, the use of chemical engineering process flow diagrams to illustrate the basic engineering principles and to facilitate the formulation of the necessary material balances was made. Third, matrix representations that enabled the formulations of the species material balances in a very compact and convenient form for presentation, record keeping, and problem solving were used [13]. Fourth, symbols with conventions that are instinctive, self-explanatory, and unambiguous were used. A set of generalized equations with few assumptions was methodically derived and then the equations were simplified under conditions generally encountered in small-scale and large-scale fire tests. Equations are expressed on amount-of-substance basis consistent with practices commonly used in stoichiometric calculations and material balances with chemical reactions.

\section{Heat Release Rate Measurements}

Figure 1 shows a schematic of a typical oxygen consumption calorimeter used to measure heat release rates. It consists of a hood under which the material of interest is burned to determine its heat release rates. All of the combustion products and the entrained air from the surroundings are drawn into the collection hood. The combustion products are sampled in the exhaust duct, and the concentrations of selected major combustion products as well as oxygen are measured after other combustion products that are not to be measured are removed by the traps. In some of the setups, a provision for the measurement of water vapor concentration in the $\mathbf{L}$-stream is also employed. The three dotted-line demarcations signify the three systems under consideration. The single $\mathbf{E}$-stream representation in Fig. 1 is a simplification; air is entrained from all sides into the burn chamber.

Figure 2 is a simplified process flow diagram representing the oxygen consumption calorimeter in Fig. 1 and the total attendant material streams for the material balances essential to the calculation of the heat release rate. Our focus will be formulating the necessary material balances on the burn chamber, the splitter, and the traps. The splitter, which divides a single input stream (the $\mathbf{L}$-stream) into two or more output streams (the $\mathbf{S}$-stream and the $\mathbf{D}$-stream) with the same composition [14], is used to represent the 
sampling process in the exhaust duct. The traps could be viewed as a separator, which separates or removes one or more components from the incoming $\mathbf{S}$-stream before exiting as the $\mathbf{A}$-stream with fewer components [14]. The components that are not removed by the separator are termed tie components in the $\mathbf{S}$-stream and the $\mathbf{A}$-stream. A tie component is defined as material that goes from one stream into another without changing in any respect or having like material added to it or lost from it [14]. The concept and identification of tie components, which are used extensively in material balance calculations in chemical engineering processes, will provide a very straightforward, convenient, and systematic procedure with which to formulate the equations needed for heat release rate calculations and will greatly facilitate solving the material balances. In the following discussion, it should be noted that the concentrations in the Astream and the $\mathbf{W}$-stream are measured and considered known. Simply put, the essence of the formulation of heat release calculations is to express the conditions in the $\mathbf{E}$-stream and the $\mathbf{L}$-stream in terms of those in the $\mathbf{A}$-stream and the $\mathbf{W}$-stream. The connection is made through the $\mathbf{S}$-stream. The reason to develop the appropriate formulas is that the presence of the traps alters the composition of the $\mathbf{S}$-stream by removing certain species from the $\mathbf{S}$-stream before the gas concentration measurements in the $\mathbf{A}$-stream. ${ }^{1}$

Based on the oxygen consumption principle, the heat release rate $q$ is defined as

$$
q=E M_{\mathrm{O}_{2}} n_{\mathrm{O}_{2}, \mathrm{rxn}}=E M_{\mathrm{O}_{2}}\left(n_{\mathrm{O}_{2}, \mathrm{E}}-n_{\mathrm{O}_{2}, \mathrm{~L}}\right),
$$

where $E=13.1 \times 10^{6} \mathrm{~J} / \mathrm{kg} \mathrm{O}_{2}$ consumed. Corrections to $E$ can be made to accommodate incomplete combustion and other conditions $[6,7,10,11]$.

The oxygen depleting factor $\phi$ was defined by Parker [6] as

$$
\phi=\frac{n_{\mathrm{O}_{2}, \mathrm{E}}-n_{\mathrm{O}_{2}, \mathrm{~L}}}{n_{\mathrm{O}_{2}, \mathrm{E}}}=\frac{n_{\mathrm{O}_{2}, \mathrm{rxn}}}{n_{\mathrm{O}_{2}, \mathrm{E}}}
$$

and

$$
\phi=\frac{n_{\mathrm{T}, \mathrm{E}} y_{\mathrm{O}_{2}, \mathrm{E}}-n_{\mathrm{T}, \mathrm{L}} y_{\mathrm{O}_{2}, \mathrm{~L}}}{n_{\mathrm{T}, \mathrm{E}} y_{\mathrm{O}_{2}, \mathrm{E}}}=1-\frac{n_{\mathrm{T}, \mathrm{L}} y_{\mathrm{O}_{2}, \mathrm{~L}}}{n_{\mathrm{T}, \mathrm{E}} y_{\mathrm{O}_{2}, \mathrm{E}}}
$$

Note that $\phi$ is equivalent to the term conversion of a reactant, used in chemical reaction engineering [15], where the reactant is oxygen (not fuel) in this case. The conversion of a reactant in a chemical reacting flow system is simply defined as the amount of a reactant reacted or consumed divided by the amount of the reactant fed to the system.

Using the definition of $\phi$, Eq. (1) can be rewritten as

$$
q=E M_{\mathrm{O}_{2}} n_{\mathrm{O}_{2}, \mathrm{E}} \phi=E M_{\mathrm{O}_{2}} y_{\mathrm{O}_{2}, \mathrm{E}} n_{\mathrm{T}, \mathrm{E}} \phi
$$

If $y_{\mathrm{O}_{2}, \mathrm{E}}, n_{\mathrm{T}, \mathrm{E}}$, and $\phi$ are known, the calculation of $q$ is very straightforward using Eq. (4). However, measuring $n_{\mathrm{T}, \mathrm{E}}$ proves to be difficult for an open system used in fire testing. One needs to come up with a means to bypass the use of $n_{\mathrm{T}, \mathrm{E}}$ in the calculations of $q$ and to relate it to $n_{\mathrm{T}, \mathrm{L}}$, which can be easily measured in the exhaust duct. What follows will be the discussion on how these three parameters $\left(y_{\mathrm{O}_{2}, \mathrm{E}}\right.$, $n_{\mathrm{T}, \mathrm{E}}$, and $\phi$ ) can be obtained from a set of formulas derived using material balances and various gas measurement schemes commonly used in fire tests.

\footnotetext{
${ }^{1}$ If no traps were present and oxygen concentration were to be measured hypothetically without the traps, the formulas derived herein for various gas measurement schemes would no longer be required because the species concentrations in the $\mathbf{L}$-stream, in the $\mathbf{S}$ stream, and in the A-stream would all be identical.
} 


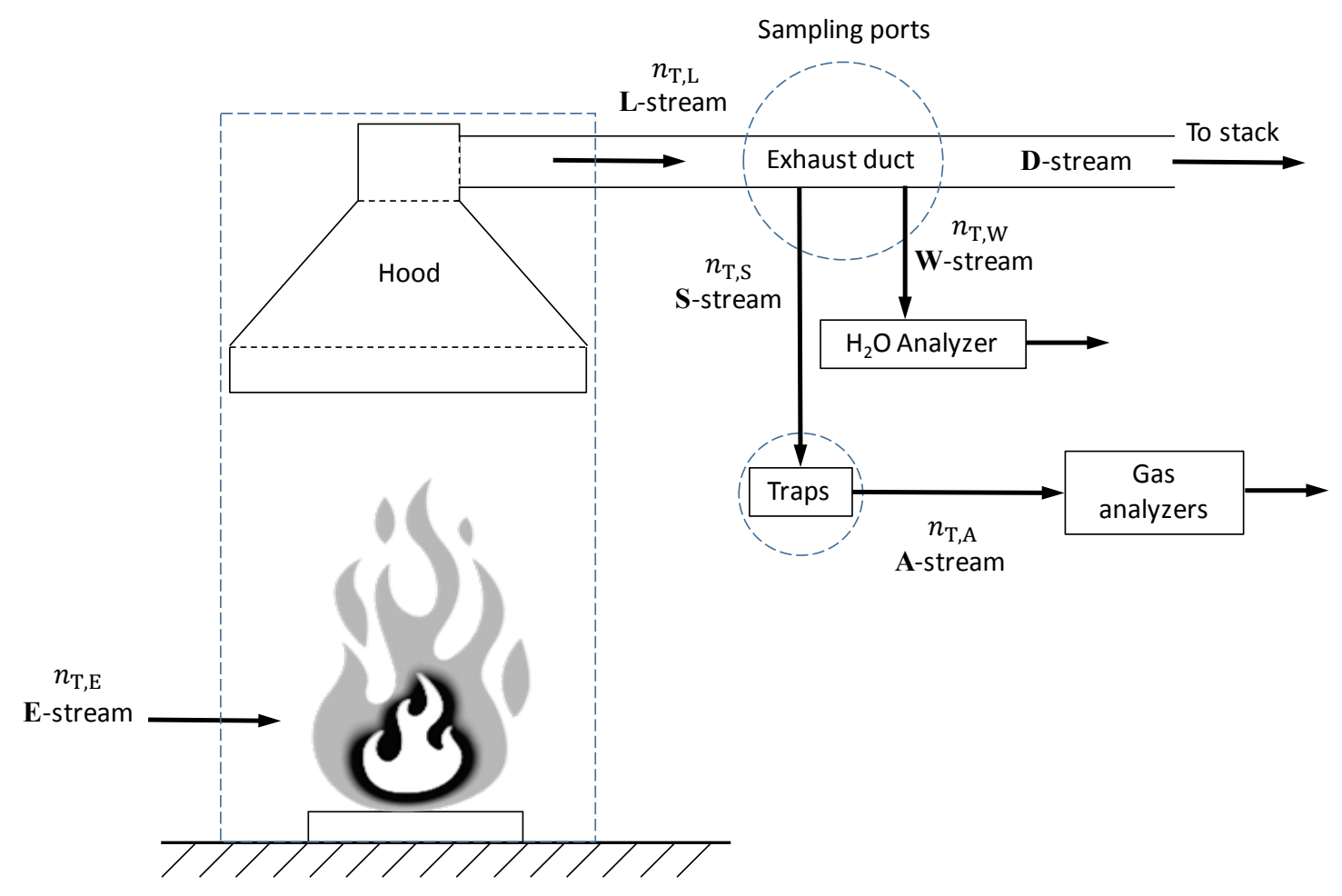

Fig. 1. Schematic of an oxygen consumption calorimeter.

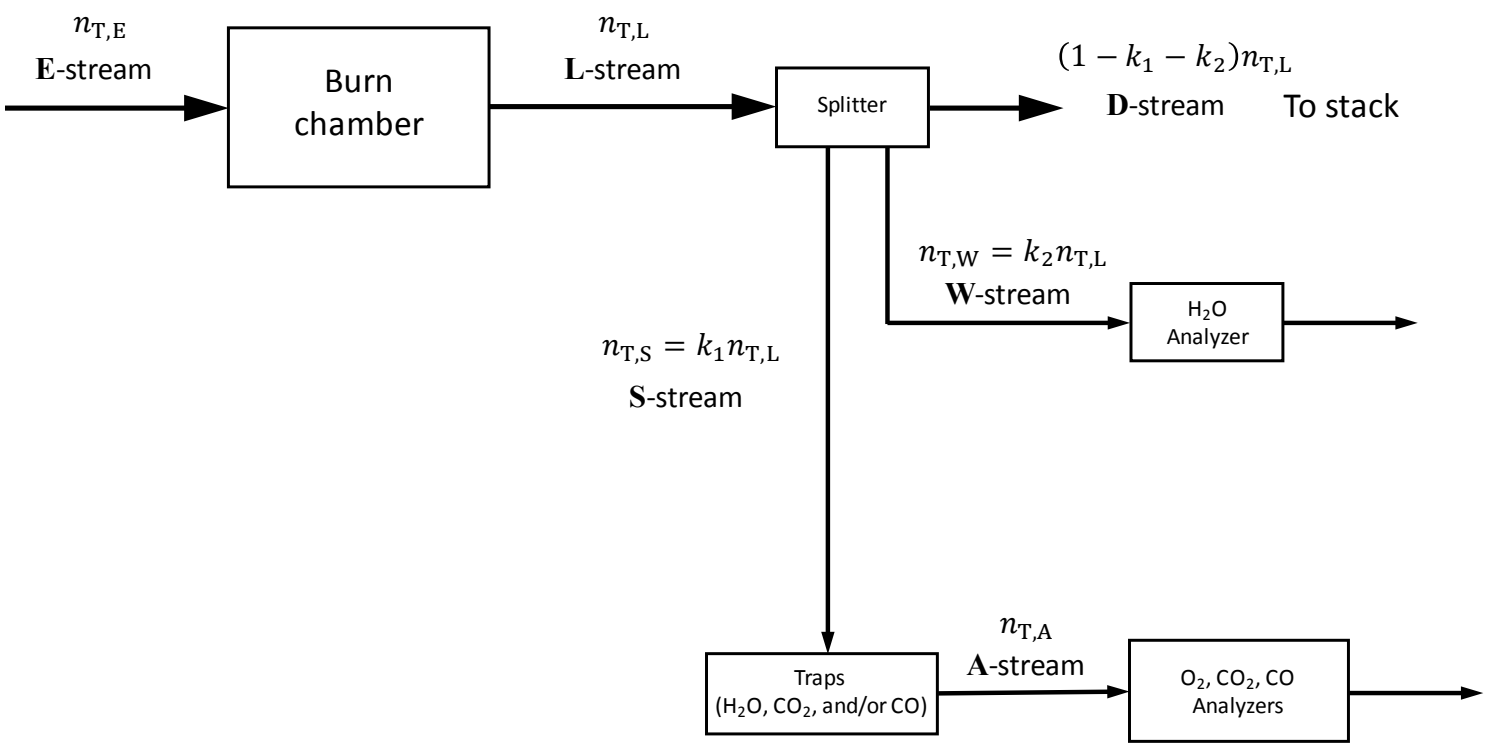

Fig. 2. Process flow diagram used for material balances. 


\section{The Essentials}

\subsection{Stoichiometry}

A general one-step global chemical reaction with the following combustion products is assumed to represent the burning occurring in the burn chamber.

$$
v_{\text {fuel }} \text { Fuel }+v_{\mathrm{O}_{2}} \mathrm{O}_{2} \rightarrow v_{\mathrm{N}_{2}} \mathrm{~N}_{2}+v_{\mathrm{CO}_{2}} \mathrm{CO}_{2}+v_{\mathrm{H}_{2} \mathrm{O}} \mathrm{H}_{2} \mathrm{O}+v_{\mathrm{CO}} \mathrm{CO}+\sum_{i=5}^{\mathrm{m}} v_{i} S_{i}
$$

To facilitate the discussion below, the first four $i$ values to the four major chemical species of interest that are used for conventional heat release measurements are explicitly assigned: $v_{1}=v_{\mathrm{N}_{2}}, v_{2}=v_{\mathrm{CO}_{2}}$, $v_{3}=v_{\mathrm{H}_{2} \mathrm{O}}, v_{4}=v_{\mathrm{CO}}, S_{1}=\mathrm{N}_{2}, S_{2}=\mathrm{CO}_{2}, S_{3}=\mathrm{H}_{2} \mathrm{O}$, and $S_{4}=\mathrm{CO}$. The summation term in Eq. (5) represents all the minor combustion product species resulting from burning. For generalization, that nitrogen is produced during burning is also considered.

\subsection{Material Balances on the Burn Chamber}

The E-stream is assumed to contain only $\mathrm{O}_{2}, \mathrm{~N}_{2}, \mathrm{CO}_{2}$, and $\mathrm{H}_{2} \mathrm{O}$. Therefore, the total material flow rate in the $\mathbf{E}$-stream is

$$
n_{\mathrm{T}, \mathrm{E}}=n_{\mathrm{O}_{2}, \mathrm{E}}+n_{\mathrm{N}_{2}, \mathrm{E}}+n_{\mathrm{CO}_{2}, \mathrm{E}}+n_{\mathrm{H}_{2} \mathrm{O}, \mathrm{E}}
$$

and

$$
y_{\mathrm{O}_{2}, \mathrm{E}}+y_{\mathrm{N}_{2}, \mathrm{E}}+y_{\mathrm{CO}_{2}, \mathrm{E}}+y_{\mathrm{H}_{2} \mathrm{O}, \mathrm{E}}=1
$$

The steady-state material balances for oxygen and the combustion product species ${ }^{2}$ written in matrix notation are

$$
\left[\begin{array}{c}
n_{\mathrm{O}_{2}, \mathrm{~L}} \\
n_{\mathrm{N}_{2}, \mathrm{~L}} \\
n_{\mathrm{CO}_{2}, \mathrm{~L}} \\
n_{\mathrm{H}_{2} \mathrm{O}, \mathrm{L}} \\
n_{\mathrm{CO}, \mathrm{L}} \\
n_{5, \mathrm{~L}} \\
\vdots \\
n_{\mathrm{m}, \mathrm{L}}
\end{array}\right]=\left[\begin{array}{c}
n_{\mathrm{O}_{2}, \mathrm{E}} \\
n_{\mathrm{N}_{2}, \mathrm{E}} \\
n_{\mathrm{CO}_{2}, \mathrm{E}} \\
n_{\mathrm{H}_{2} \mathrm{O}, \mathrm{E}} \\
0 \\
0 \\
\vdots \\
0
\end{array}\right]+\left[\begin{array}{c}
-n_{\mathrm{O}_{2}, \mathrm{rxn}} \\
n_{\mathrm{N}_{2}, \mathrm{rxn}} \\
n_{\mathrm{CO}_{2} \mathrm{rxn}} \\
n_{\mathrm{H}_{2} \mathrm{O}, \mathrm{rxn}} \\
n_{\mathrm{CO}, \mathrm{rxn}} \\
n_{5, \mathrm{rxn}} \\
\vdots \\
n_{\mathrm{m}, \mathrm{rxn}}
\end{array}\right]=\left[\begin{array}{c}
n_{\mathrm{O}_{2}, \mathrm{E}} \\
n_{\mathrm{N}_{2}, \mathrm{E}} \\
n_{\mathrm{CO}_{2}, \mathrm{E}} \\
n_{\mathrm{H}_{2} \mathrm{O}, \mathrm{E}} \\
0 \\
0 \\
\vdots \\
0
\end{array}\right]+\left[\begin{array}{c}
-1 \\
v_{\mathrm{N}_{2}} / v_{\mathrm{O}_{2}} \\
v_{\mathrm{CO}_{2}} / v_{\mathrm{O}_{2}} \\
v_{\mathrm{H}_{2} \mathrm{O}} / v_{\mathrm{O}_{2}} \\
v_{\mathrm{CO}} / v_{\mathrm{O}_{2}} \\
v_{5} / v_{\mathrm{O}_{2}} \\
\vdots \\
v_{\mathrm{m}} / v_{\mathrm{O}_{2}}
\end{array}\right] n_{\mathrm{O}_{2}, \mathrm{rxn}}
$$

The total material flow rate in the $\mathbf{L}$-stream is

$$
n_{\mathrm{T}, \mathrm{L}}=n_{\mathrm{O}_{2}, \mathrm{~L}}+n_{\mathrm{N}_{2}, \mathrm{~L}}+n_{\mathrm{H}_{2} \mathrm{O}, \mathrm{L}}+n_{\mathrm{CO}_{2}, \mathrm{~L}}+n_{\mathrm{CO}, \mathrm{L}}+\sum_{i=5}^{\mathrm{m}} n_{i, \mathrm{~L}}
$$

\footnotetext{
${ }^{2}$ If there is excess unburnt fuel vapor in the $\mathbf{L}$-stream, and the fuel burning rate is known, it can be easily taken into consideration in Eq. (8) by treating the unburnt fuel vapor as one of the $m$ species, with $n_{\text {fuel,E }}$ equal to the fuel burning rate and the fuel consumption rate equal to $\left(-v_{\text {fuel }} / v_{\mathrm{O}_{2}}\right) n_{\mathrm{O}_{2} \text {,rxn }}$.
} 
and

$$
y_{\mathrm{O}_{2}, \mathrm{~L}}+y_{\mathrm{N}_{2}, \mathrm{~L}}+y_{\mathrm{H}_{2} \mathrm{O}, \mathrm{L}}+y_{\mathrm{CO}_{2}, \mathrm{~L}}+y_{\mathrm{CO}, \mathrm{L}}+\sum_{i=5}^{\mathrm{m}} y_{i, \mathrm{~L}}=1
$$

Substituting the corresponding matrix elements in Eq. (8) into Eq. (9) and simplifying using Eq. (6), one obtains

$$
n_{\mathrm{T}, \mathrm{L}}=n_{\mathrm{T}, \mathrm{E}}+\left(\frac{v_{\mathrm{N}_{2}}}{v_{\mathrm{O}_{2}}}+\frac{v_{\mathrm{H}_{2} \mathrm{O}}}{v_{\mathrm{O}_{2}}}+\frac{v_{\mathrm{CO}_{2}}}{v_{\mathrm{O}_{2}}}+\frac{v_{\mathrm{CO}}}{v_{\mathrm{O}_{2}}}+\sum_{i=5}^{\mathrm{m}} \frac{v_{i}}{v_{\mathrm{O}_{2}}}-1\right)\left(y_{\mathrm{O}_{2}, \mathrm{E}} n_{\mathrm{T}, \mathrm{E}}-y_{\mathrm{O}_{2}, \mathrm{~L}} n_{\mathrm{T}, \mathrm{L}}\right)
$$

Define

$$
\beta=\left(\frac{v_{\mathrm{N}_{2}}}{v_{\mathrm{O}_{2}}}+\frac{v_{\mathrm{H}_{2} \mathrm{O}}}{v_{\mathrm{O}_{2}}}+\frac{v_{\mathrm{CO}_{2}}}{v_{\mathrm{O}_{2}}}+\frac{v_{\mathrm{CO}}}{v_{\mathrm{O}_{2}}}+\sum_{i=5}^{\mathrm{m}} \frac{v_{i}}{v_{\mathrm{O}_{2}}}\right)
$$

This definition is analogous to that defined by Parker [6]. Using Eq. (12), Eq. (11) can be rewritten as

$$
\frac{n_{\mathrm{T}, \mathrm{L}}}{n_{\mathrm{T}, \mathrm{E}}}=\frac{1+(\beta-1) y_{\mathrm{O}_{2}, \mathrm{E}}}{1+(\beta-1) y_{\mathrm{O}_{2}, \mathrm{~L}}}
$$

Using the definition of $\phi$, Eq. (13) can be expressed in terms of $\phi$ as

$$
\frac{n_{\mathrm{T}, \mathrm{L}}}{n_{\mathrm{T}, \mathrm{E}}}=1+(\beta-1) \phi y_{\mathrm{O}_{2}, \mathrm{E}}
$$

Following Parker [6], the expansion factor $\alpha$ can be defined as the ratio of $n_{\mathrm{T}, \mathrm{L}}$ to $n_{\mathrm{T}, \mathrm{E}}$ with $n_{\mathrm{T}, \mathrm{L}}$ completely depleted of its oxygen (i.e., $y_{\mathrm{O}_{2}, \mathrm{~L}}=0$ ). From Eq. (13) with $y_{\mathrm{O}_{2}, \mathrm{~L}}=0$, one obtains

$$
\alpha=\frac{\left|n_{\mathrm{T}, \mathrm{L}}\right|_{y_{\mathrm{O}_{2}, \mathrm{~L}}=0}}{n_{\mathrm{T}, \mathrm{E}}}=\frac{1+(\beta-1) y_{\mathrm{O}_{2}, \mathrm{E}}}{1+(\beta-1) y_{\mathrm{O}_{2}, \mathrm{~L}}}=1+(\beta-1) y_{\mathrm{O}_{2}, \mathrm{E}}
$$

Substituting Eq. (15) into Eq. (14),

$$
\frac{n_{\mathrm{T}, \mathrm{E}}}{n_{\mathrm{T}, \mathrm{L}}}=\frac{1}{1+(\alpha-1) \phi}
$$

Therefore, the material balances on the burner chamber provide a relationship between $n_{\mathrm{T}, \mathrm{E}}$ and $n_{\mathrm{T}, \mathrm{L}}$ in terms of $\beta$ and $\phi$ [Eq. (14)] or $\alpha$ and $\phi$ [Eq. (16)]. If water vapor is measured in the $\mathbf{L}$-stream, then $\beta$ can be obtained from the procedure described in Appendix B; otherwise, a nominal value of 1.1 for $\alpha$ is recommended [6], which is approximately the value for methane.

\subsection{Material Balances on the Splitter}

The splitter signifies the process occurring at the sampling port in the exhaust duct. By the physical nature of sampling, the species concentrations in the $\mathbf{L}$-stream, the $\mathbf{S}$-stream, the $\mathbf{W}$-stream, and the $\mathbf{D}$ stream are the same. 


$$
\left[\begin{array}{c}
y_{\mathrm{O}_{2}, \mathrm{~S}} \\
y_{\mathrm{N}_{2}, \mathrm{~S}} \\
y_{\mathrm{CO}_{2}, \mathrm{~S}} \\
y_{\mathrm{H}_{2} \mathrm{O}, \mathrm{S}} \\
y_{\mathrm{CO}, \mathrm{S}} \\
y_{5, \mathrm{~S}} \\
y_{6, \mathrm{~S}} \\
\vdots \\
y_{\mathrm{m}, \mathrm{S}}
\end{array}\right]=\left[\begin{array}{c}
y_{\mathrm{O}_{2}, \mathrm{~L}} \\
y_{\mathrm{N}_{2}, \mathrm{~L}} \\
y_{\mathrm{CO}_{2}, \mathrm{~L}} \\
y_{\mathrm{H}_{2} \mathrm{O}, \mathrm{L}} \\
y_{\mathrm{CO}, \mathrm{L}} \\
y_{5, \mathrm{~L}} \\
y_{6, \mathrm{~L}} \\
\vdots \\
y_{\mathrm{m}, \mathrm{L}}
\end{array}\right]=\left[\begin{array}{c}
y_{\mathrm{O}_{2}, \mathrm{~W}} \\
y_{\mathrm{N}_{2}, \mathrm{~W}} \\
y_{\mathrm{CO}_{2}, \mathrm{~W}} \\
y_{\mathrm{H}_{2} \mathrm{O}, \mathrm{W}} \\
y_{\mathrm{CO}, \mathrm{W}} \\
y_{5, \mathrm{~W}} \\
y_{6, \mathrm{~W}} \\
\vdots \\
y_{\mathrm{m}, \mathrm{W}}
\end{array}\right]=\left[\begin{array}{c}
y_{\mathrm{O}_{2}, \mathrm{D}} \\
y_{\mathrm{N}_{2}, \mathrm{D}} \\
y_{\mathrm{CO}_{2}, \mathrm{D}} \\
y_{\mathrm{H}_{2} \mathrm{O}, \mathrm{D}} \\
y_{\mathrm{CO}, \mathrm{D}} \\
y_{5, \mathrm{D}} \\
y_{6, \mathrm{D}} \\
\vdots \\
y_{\mathrm{m}, \mathrm{D}}
\end{array}\right]
$$

Then,

$$
\frac{1}{n_{\mathrm{T}, \mathrm{S}}}\left[\begin{array}{c}
n_{\mathrm{O}_{2}, \mathrm{~S}} \\
n_{\mathrm{N}_{2}, \mathrm{~S}} \\
n_{\mathrm{CO}_{2}, \mathrm{~S}} \\
n_{\mathrm{H}_{2} \mathrm{O}, \mathrm{S}} \\
n_{\mathrm{CO}, \mathrm{S}} \\
n_{5, \mathrm{~S}} \\
n_{6, \mathrm{~S}} \\
\vdots \\
n_{\mathrm{m}, \mathrm{S}}
\end{array}\right]=\frac{1}{n_{\mathrm{T}, \mathrm{L}}}\left[\begin{array}{c}
n_{\mathrm{O}_{2}, \mathrm{~L}} \\
n_{\mathrm{N}_{2}, \mathrm{~L}} \\
n_{\mathrm{CO}_{2}, \mathrm{~L}} \\
n_{\mathrm{H}_{2} \mathrm{O}, \mathrm{L}} \\
n_{\mathrm{CO}, \mathrm{L}} \\
n_{5, \mathrm{~L}} \\
n_{6, \mathrm{~L}} \\
\vdots \\
n_{\mathrm{m}, \mathrm{L}}
\end{array}\right]=\frac{1}{n_{\mathrm{T}, \mathrm{W}}}\left[\begin{array}{c}
n_{\mathrm{O}_{2}, \mathrm{~W}} \\
n_{\mathrm{N}_{2}, \mathrm{~W}} \\
n_{\mathrm{CO}_{2}, \mathrm{~W}} \\
n_{\mathrm{H}_{2} \mathrm{O}, \mathrm{W}} \\
n_{\mathrm{CO}, \mathrm{W}} \\
n_{5, \mathrm{~W}} \\
n_{6, \mathrm{~W}} \\
\vdots \\
n_{\mathrm{m}, \mathrm{W}}
\end{array}\right]=\frac{1}{n_{\mathrm{T}, \mathrm{D}}}\left[\begin{array}{c}
n_{\mathrm{O}_{2}, \mathrm{D}} \\
n_{\mathrm{N}_{2}, \mathrm{D}} \\
n_{\mathrm{CO}_{2}, \mathrm{D}} \\
n_{\mathrm{H}_{2} \mathrm{O}, \mathrm{D}} \\
n_{\mathrm{CO}, \mathrm{D}} \\
n_{5, \mathrm{D}} \\
n_{6, \mathrm{D}} \\
\vdots \\
n_{\mathrm{m}, \mathrm{D}}
\end{array}\right]
$$

and

$$
\left[\begin{array}{c}
n_{\mathrm{O}_{2}, \mathrm{~S}} \\
n_{\mathrm{N}_{2}, \mathrm{~S}} \\
n_{\mathrm{CO}_{2}, \mathrm{~S}} \\
n_{\mathrm{H}_{2} \mathrm{O}, \mathrm{S}} \\
n_{\mathrm{CO}, \mathrm{S}} \\
n_{5, \mathrm{~S}} \\
n_{6, \mathrm{~S}} \\
\vdots \\
n_{\mathrm{m}, \mathrm{S}}
\end{array}\right]=\frac{n_{\mathrm{T}, \mathrm{S}}}{n_{\mathrm{T}, \mathrm{L}}}\left[\begin{array}{c}
n_{\mathrm{O}_{2}, \mathrm{~L}} \\
n_{\mathrm{N}_{2}, \mathrm{~L}} \\
n_{\mathrm{CO}_{2} \mathrm{~L}} \\
n_{\mathrm{H}_{2} \mathrm{O}, \mathrm{L}} \\
n_{\mathrm{CO}, \mathrm{L}} \\
n_{5, \mathrm{~L}} \\
n_{6, \mathrm{~L}} \\
\vdots \\
n_{\mathrm{m}, \mathrm{L}}
\end{array}\right]=\frac{n_{\mathrm{T}, \mathrm{S}}}{n_{\mathrm{T}, \mathrm{W}}}\left[\begin{array}{c}
n_{\mathrm{O}_{2}, \mathrm{~W}} \\
n_{\mathrm{N}_{2}, \mathrm{~W}} \\
n_{\mathrm{CO}_{2}, \mathrm{~W}} \\
n_{\mathrm{H}_{2} \mathrm{O}, \mathrm{W}} \\
n_{\mathrm{CO}, \mathrm{W}} \\
n_{5, \mathrm{~W}} \\
n_{6, \mathrm{~W}} \\
\vdots \\
n_{\mathrm{m}, \mathrm{W}}
\end{array}\right]=\frac{n_{\mathrm{T}, \mathrm{S}}}{n_{\mathrm{T}, \mathrm{D}}}\left[\begin{array}{c}
n_{\mathrm{O}_{2}, \mathrm{D}} \\
n_{\mathrm{N}_{2}, \mathrm{D}} \\
n_{\mathrm{CO}_{2}, \mathrm{D}} \\
n_{\mathrm{H}_{2} \mathrm{O}, \mathrm{D}} \\
n_{\mathrm{CO}, \mathrm{D}} \\
n_{5, \mathrm{D}} \\
n_{6, \mathrm{D}} \\
\vdots \\
n_{\mathrm{m}, \mathrm{D}}
\end{array}\right] .
$$

Since $n_{\mathrm{T}, \mathrm{L}}=n_{\mathrm{T}, \mathrm{S}}+n_{\mathrm{T}, \mathrm{W}}+n_{\mathrm{T}, \mathrm{D}}$, Eq. (18) becomes

$$
\left[\begin{array}{c}
n_{\mathrm{O}_{2}, \mathrm{~S}} \\
n_{\mathrm{N}_{2}, \mathrm{~S}} \\
n_{\mathrm{CO}_{2}, \mathrm{~S}} \\
n_{\mathrm{H}_{2} \mathrm{O}, \mathrm{S}} \\
n_{\mathrm{CO}, \mathrm{S}} \\
n_{5, \mathrm{~S}} \\
n_{6, \mathrm{~S}} \\
\vdots \\
n_{\mathrm{m}, \mathrm{S}}
\end{array}\right]=\frac{n_{\mathrm{T}, \mathrm{S}}}{n_{\mathrm{T}, \mathrm{L}}}\left[\begin{array}{c}
n_{\mathrm{O}_{2}, \mathrm{~L}} \\
n_{\mathrm{N}_{2}, \mathrm{~L}} \\
n_{\mathrm{CO}_{2}, \mathrm{~L}} \\
n_{\mathrm{H}_{2} \mathrm{O}, \mathrm{L}} \\
n_{\mathrm{CO}, \mathrm{L}} \\
n_{5, \mathrm{~L}} \\
n_{6, \mathrm{~L}} \\
\vdots \\
n_{\mathrm{m}, \mathrm{L}}
\end{array}\right]=\frac{n_{\mathrm{T}, \mathrm{S}}}{n_{\mathrm{T}, \mathrm{W}}}\left[\begin{array}{c}
n_{\mathrm{O}_{2}, \mathrm{~W}} \\
n_{\mathrm{N}_{2}, \mathrm{~W}} \\
n_{\mathrm{CO}_{2}, \mathrm{~W}} \\
n_{\mathrm{H}_{2} \mathrm{O}, \mathrm{W}} \\
n_{\mathrm{CO}, \mathrm{W}} \\
n_{5, \mathrm{~W}} \\
n_{6, \mathrm{~W}} \\
\vdots \\
n_{\mathrm{m}, \mathrm{W}}
\end{array}\right]=\frac{n_{\mathrm{T}, \mathrm{S}}}{n_{\mathrm{T}, \mathrm{L}}-n_{\mathrm{T}, \mathrm{S}}-n_{\mathrm{T}, \mathrm{W}}}\left[\begin{array}{c}
n_{\mathrm{O}_{2}, \mathrm{D}} \\
n_{\mathrm{N}_{2}, \mathrm{D}} \\
n_{\mathrm{CO}_{2}, \mathrm{D}} \\
n_{\mathrm{H}_{2} \mathrm{O}, \mathrm{D}} \\
n_{\mathrm{CO}, \mathrm{D}} \\
n_{5, \mathrm{D}} \\
n_{6, \mathrm{D}} \\
\vdots \\
n_{\mathrm{m}, \mathrm{D}}
\end{array}\right] .
$$

Define

$$
k_{1}=\frac{n_{\mathrm{T}, \mathrm{S}}}{n_{\mathrm{T}, \mathrm{L}}},
$$

and

$$
k_{2}=\frac{n_{\mathrm{T}, \mathrm{W}}}{n_{\mathrm{T}, \mathrm{L}}}
$$


Equation (19) becomes

$$
\left[\begin{array}{c}
n_{\mathrm{O}_{2}, \mathrm{~S}} \\
n_{\mathrm{N}_{2}, \mathrm{~S}} \\
n_{\mathrm{CO}_{2}, \mathrm{~S}} \\
n_{\mathrm{H}_{2} \mathrm{O}, \mathrm{S}} \\
n_{\mathrm{CO}, \mathrm{S}} \\
n_{5, \mathrm{~S}} \\
n_{6, \mathrm{~S}} \\
\vdots \\
n_{\mathrm{m}, \mathrm{S}}
\end{array}\right]=k_{1}\left[\begin{array}{c}
n_{\mathrm{O}_{2}, \mathrm{~L}} \\
n_{\mathrm{N}_{2}, \mathrm{~L}} \\
n_{\mathrm{CO}_{2} \mathrm{~L}} \\
n_{\mathrm{H}_{2} \mathrm{O}, \mathrm{L}} \\
n_{\mathrm{CO}, \mathrm{L}} \\
n_{5, \mathrm{~L}} \\
n_{6, \mathrm{~L}} \\
\vdots \\
n_{\mathrm{m}, \mathrm{L}}
\end{array}\right]=\frac{k_{1}}{k_{2}}\left[\begin{array}{c}
n_{\mathrm{O}_{2}, \mathrm{~W}} \\
n_{\mathrm{N}_{2}, \mathrm{~W}} \\
n_{\mathrm{CO}_{2}, \mathrm{~W}} \\
n_{\mathrm{H}_{2} \mathrm{O}, \mathrm{W}} \\
n_{\mathrm{CO}, \mathrm{W}} \\
n_{5, \mathrm{~W}} \\
n_{6, \mathrm{~W}} \\
\vdots \\
n_{\mathrm{m}, \mathrm{W}}
\end{array}\right]=\frac{k_{1}}{1-k_{1}-k_{2}}\left[\begin{array}{c}
n_{\mathrm{O}_{2}, \mathrm{D}} \\
n_{\mathrm{N}_{2}, \mathrm{D}} \\
n_{\mathrm{CO}_{2}, \mathrm{D}} \\
n_{\mathrm{H}_{2} \mathrm{O}, \mathrm{D}} \\
n_{\mathrm{CO}, \mathrm{D}} \\
n_{5, \mathrm{D}} \\
n_{6, \mathrm{D}} \\
\vdots \\
n_{\mathrm{m}, \mathrm{D}}
\end{array}\right] .
$$

For gas sampling, $k_{1} \ll 1, k_{2} \ll 1$, and $n_{\mathrm{T}, \mathrm{S}}$ (the $\mathbf{S}$-stream) and $n_{\mathrm{T}, \mathrm{W}}$ (the $\mathbf{W}$-stream) are only a very small fraction of $n_{\mathrm{T}, \mathrm{L}}$ (the L-stream), and $n_{\mathrm{T}, \mathrm{D}} \gg n_{\mathrm{T}, \mathrm{S}}+n_{\mathrm{T}, \mathrm{W}}$.

\subsection{Material Balances on the Traps}

In most oxygen-consumption calorimeters used to measure heat release rates, only the major species, i.e., $\mathrm{O}_{2}, \mathrm{CO}_{2}, \mathrm{H}_{2} \mathrm{O}$, and/or $\mathrm{CO}$, are measured by the gas analyzers. The discussion below assumes $d r y$-basis measurements for $\mathrm{O}_{2}, \mathrm{CO}_{2}$, and $\mathrm{CO}$. In most fire-test applications, one of the following four specific gas measurement schemes (schemes A, B, C, and D) will typically be employed, depending on the species that is/are removed by the traps.

\subsubsection{Scheme A (O2 Measured)}

In this case, $\mathrm{H}_{2} \mathrm{O}, \mathrm{CO}_{2}$, and $\mathrm{CO}$ are removed by the traps, and $\mathrm{O}_{2}, \mathrm{~N}_{2}$, and $S_{i}(i=5 \ldots \mathrm{m})$ are the tie components in the $\mathbf{S}$-stream and the $\mathbf{A}$-stream. One has

$$
\begin{gathered}
n_{\mathrm{T}, \mathrm{W}}=0, \\
n_{\mathrm{T}, \mathrm{A}}=n_{\mathrm{O}_{2}, \mathrm{~A}}+n_{\mathrm{N}_{2}, \mathrm{~A}}+\sum_{i=5}^{\mathrm{m}} n_{i, \mathrm{~A}}, \\
n_{\mathrm{H}_{2} \mathrm{O}, \mathrm{A}}=n_{\mathrm{CO}_{2}, \mathrm{~A}}=n_{\mathrm{CO}, \mathrm{A}}=0, \\
y_{\mathrm{O}_{2}, \mathrm{~A}}+y_{\mathrm{N}_{2}, \mathrm{~A}}+\sum_{i=5}^{\mathrm{m}} y_{i, \mathrm{~A}}=1,
\end{gathered}
$$

and

$$
\left[\begin{array}{c}
n_{\mathrm{O}_{2}, \mathrm{~S}} \\
n_{\mathrm{N}_{2}, \mathrm{~S}} \\
n_{5, \mathrm{~S}} \\
\vdots \\
n_{\mathrm{m}, \mathrm{S}}
\end{array}\right]=\left[\begin{array}{c}
n_{\mathrm{O}_{2}, \mathrm{~A}} \\
n_{\mathrm{N}_{2}, \mathrm{~A}} \\
n_{5, \mathrm{~A}} \\
\vdots \\
n_{\mathrm{m}, \mathrm{A}}
\end{array}\right]
$$

Using Eq. (22), Eq. (25) becomes 


$$
\left[\begin{array}{c}
n_{\mathrm{O}_{2}, \mathrm{~L}} \\
n_{\mathrm{N}_{2}, \mathrm{~L}} \\
n_{5, \mathrm{~L}} \\
\vdots \\
n_{\mathrm{m}, \mathrm{L}}
\end{array}\right]=\frac{1}{k_{1}}\left[\begin{array}{c}
n_{\mathrm{O}_{2}, \mathrm{~A}} \\
n_{\mathrm{N}_{2}, \mathrm{~A}} \\
n_{5, \mathrm{~A}} \\
\vdots \\
n_{\mathrm{m}, \mathrm{A}}
\end{array}\right]
$$

and

$$
n_{\mathrm{T}, \mathrm{L}}\left[\begin{array}{c}
y_{\mathrm{O}_{2}, \mathrm{~L}} \\
y_{\mathrm{N}_{2} \mathrm{~L}} \\
y_{5, \mathrm{~L}} \\
\vdots \\
y_{\mathrm{m}, \mathrm{L}}
\end{array}\right]=\frac{n_{\mathrm{T}, \mathrm{A}}}{k_{1}}\left[\begin{array}{c}
y_{\mathrm{O}_{2}, \mathrm{~A}} \\
y_{\mathrm{N}_{2}, \mathrm{~A}} \\
y_{5, \mathrm{~A}} \\
\vdots \\
y_{\mathrm{m}, \mathrm{A}}
\end{array}\right]
$$

Using Eq. (23),

$$
\left[\begin{array}{c}
y_{\mathrm{O}_{2}, \mathrm{~L}} \\
y_{\mathrm{N}_{2}, \mathrm{~L}} \\
y_{5, \mathrm{~L}} \\
\vdots \\
y_{\mathrm{m}, \mathrm{L}}
\end{array}\right]=\frac{n_{\mathrm{O}_{2}, \mathrm{~A}}+n_{\mathrm{N}_{2}, \mathrm{~A}}+\sum_{i=5}^{\mathrm{m}} n_{i, \mathrm{~A}}}{k_{1} n_{\mathrm{T}, \mathrm{L}}}\left[\begin{array}{c}
y_{\mathrm{O}_{2}, \mathrm{~A}} \\
y_{\mathrm{N}_{2}, \mathrm{~A}} \\
y_{5, \mathrm{~A}} \\
\vdots \\
y_{\mathrm{m}, \mathrm{A}}
\end{array}\right]
$$

Using Eq. (22) and Eq. (25), it can be easily shown that

$$
\left[\begin{array}{c}
y_{\mathrm{O}_{2}, \mathrm{~L}} \\
y_{\mathrm{N}_{2}, \mathrm{~L}} \\
y_{5, \mathrm{~L}} \\
\vdots \\
y_{\mathrm{m}, \mathrm{L}}
\end{array}\right]=\left(y_{\mathrm{O}_{2}, \mathrm{~L}}+y_{\mathrm{N}_{2}, \mathrm{~L}}+\sum_{i=5}^{\mathrm{m}} y_{i, \mathrm{~L}}\right)\left[\begin{array}{c}
y_{\mathrm{O}_{2}, \mathrm{~A}} \\
y_{\mathrm{N}_{2}, \mathrm{~A}} \\
y_{5, \mathrm{~A}} \\
\vdots \\
y_{\mathrm{m}, \mathrm{A}}
\end{array}\right] .
$$

Using Eq. (10), Eq. (26) can be expressed as

$$
\left[\begin{array}{c}
y_{\mathrm{O}_{2}, \mathrm{~L}} \\
y_{\mathrm{N}_{2}, \mathrm{~L}} \\
y_{5, \mathrm{~L}} \\
\vdots \\
y_{\mathrm{m}, \mathrm{L}}
\end{array}\right]=\left(1-y_{\mathrm{H}_{2} \mathrm{O}, \mathrm{L}}-y_{\mathrm{CO}_{2}, \mathrm{~L}}-y_{\mathrm{CO}, \mathrm{L}}\right)\left[\begin{array}{c}
y_{\mathrm{O}_{2}, \mathrm{~A}} \\
y_{\mathrm{N}_{2}, \mathrm{~A}} \\
y_{5, \mathrm{~A}} \\
\vdots \\
y_{\mathrm{m}, \mathrm{A}}
\end{array}\right] .
$$

Equation (26) can be rewritten as

$$
\left[\begin{array}{ccccccc}
\left(1-y_{\mathrm{O}_{2}, \mathrm{~A}}\right) & -y_{\mathrm{O}_{2}, \mathrm{~A}} & -y_{\mathrm{O}_{2}, \mathrm{~A}} & -y_{\mathrm{O}_{2}, \mathrm{~A}} & -y_{\mathrm{O}_{2}, \mathrm{~A}} & \cdots & -y_{\mathrm{O}_{2}, \mathrm{~A}} \\
-y_{\mathrm{N}_{2}, \mathrm{~A}} & \left(1-y_{\mathrm{N}_{2}, \mathrm{~A}}\right) & -y_{\mathrm{N}_{2}, \mathrm{~A}} & -y_{\mathrm{N}_{2}, \mathrm{~A}} & -y_{\mathrm{N}_{2}, \mathrm{~A}} & \cdots & -y_{\mathrm{N}_{2}, \mathrm{~A}} \\
-y_{5, \mathrm{~A}} & -y_{5, \mathrm{~A}} & \left(1-y_{5, \mathrm{~A}}\right) & -y_{5, \mathrm{~A}} & -y_{5, \mathrm{~A}} & \cdots & -y_{5, \mathrm{~A}} \\
\vdots & \vdots & \vdots & \vdots & \vdots & \ddots & \vdots \\
-y_{\mathrm{m}, \mathrm{A}} & -y_{\mathrm{m}, \mathrm{A}} & -y_{\mathrm{m}, \mathrm{A}} & -y_{\mathrm{m}, \mathrm{A}} & -y_{\mathrm{m}, \mathrm{A}} & \cdots & \left(1-y_{\mathrm{m}, \mathrm{A}}\right)
\end{array}\right]\left[\begin{array}{c}
y_{\mathrm{O}_{2}, \mathrm{~L}} \\
y_{\mathrm{N}_{2}, \mathrm{~L}} \\
y_{5, \mathrm{~L}} \\
\vdots \\
y_{\mathrm{m}, \mathrm{L}}
\end{array}\right]=\left[\begin{array}{c}
0 \\
0 \\
0 \\
\vdots \\
0
\end{array}\right] .
$$

The above $(m-2) \times(m-2)$ coefficient matrix has a rank of $(m-3)$; therefore, only $(m-3)$ equations are independent [16]. One corresponding row from the three matrices in Eq. (28) may be omitted and only the matrices with the rows associated with $y_{\mathrm{O}_{2}, \mathrm{~L}}$, and $y_{5, \mathrm{~L}} \cdots y_{\mathrm{m}, \mathrm{L}}$ is considered. Equation (28) can be manipulated and reduced to the following form. 


$$
\left[\begin{array}{cccc}
\left(1-y_{\mathrm{O}_{2}, \mathrm{~A}}\right) & -y_{\mathrm{O}_{2}, \mathrm{~A}} & \cdots & -y_{\mathrm{O}_{2}, \mathrm{~A}} \\
-y_{5, \mathrm{~A}} & \left(1-y_{5, \mathrm{~A}}\right) & \cdots & -y_{5, \mathrm{~A}} \\
\vdots & \vdots & \ddots & \vdots \\
-y_{\mathrm{m}, \mathrm{A}} & -y_{\mathrm{m}, \mathrm{A}} & \cdots & \left(1-y_{\mathrm{m}, \mathrm{A}}\right)
\end{array}\right]\left[\begin{array}{c}
y_{\mathrm{O}_{2}, \mathrm{~L}} \\
y_{5, \mathrm{~L}} \\
\vdots \\
y_{\mathrm{m}, \mathrm{L}}
\end{array}\right]=\left[\begin{array}{cc}
y_{\mathrm{O}_{2}, \mathrm{~A}} & y_{\mathrm{N}_{2}, \mathrm{~L}} \\
y_{5, \mathrm{~A}} & y_{\mathrm{N}_{2}, \mathrm{~L}} \\
\vdots \\
y_{\mathrm{m}, \mathrm{A}} y_{\mathrm{N}_{2}, \mathrm{~L}}
\end{array}\right]
$$

Solving for $y_{\mathrm{O}_{2}, \mathrm{~L}}$ and $y_{5, \mathrm{~L}} \cdots y_{\mathrm{m}, \mathrm{L}}$,

$$
\left[\begin{array}{c}
y_{\mathrm{O}_{2}, \mathrm{~L}} \\
y_{5, \mathrm{~L}} \\
\vdots \\
y_{\mathrm{m}, \mathrm{L}}
\end{array}\right]=\left[\begin{array}{cccc}
\left(1-y_{\mathrm{O}_{2}, \mathrm{~A}}\right) & -y_{\mathrm{O}_{2}, \mathrm{~A}} & \cdots & -y_{\mathrm{O}_{2}, \mathrm{~A}} \\
-y_{5, \mathrm{~A}} & \left(1-y_{5, \mathrm{~A}}\right) & \cdots & -y_{5, \mathrm{~A}} \\
\vdots & \vdots & \ddots & \vdots \\
-y_{\mathrm{m}, \mathrm{A}} & -y_{\mathrm{m}, \mathrm{A}} & \cdots & \left(1-y_{\mathrm{m}, \mathrm{A}}\right)
\end{array}\right]^{-1}\left[\begin{array}{c}
y_{\mathrm{O}_{2}, \mathrm{~A}} y_{\mathrm{N}_{2}, \mathrm{~L}} \\
y_{5, \mathrm{~A}} y_{\mathrm{N}_{2}, \mathrm{~L}} \\
\vdots \\
y_{\mathrm{m}, \mathrm{A}} y_{\mathrm{N}_{2}, \mathrm{~L}}
\end{array}\right]
$$

Simplifying by using the inverse of the above matrix given in Appendix A, Eq. (30) becomes

$$
\left[\begin{array}{c}
y_{\mathrm{O}_{2}, \mathrm{~L}} \\
y_{5, \mathrm{~L}} \\
\vdots \\
y_{\mathrm{m}, \mathrm{L}}
\end{array}\right]=\frac{y_{\mathrm{N}_{2}, \mathrm{~L}}}{\left(1-y_{\mathrm{O}_{2}, \mathrm{~A}}-\sum_{i=5}^{\mathrm{m}} y_{i, \mathrm{~A}}\right)}\left[\begin{array}{c}
y_{\mathrm{O}_{2, \mathrm{~A}} \mathrm{~A}} \\
y_{5, \mathrm{~A}} \\
\vdots \\
y_{\mathrm{m}, \mathrm{A}}
\end{array}\right] \text {. }
$$

In most fire test applications, the major combustion product species in the $\mathbf{L}$-stream are $\mathrm{O}_{2}, \mathrm{~N}_{2}, \mathrm{CO}_{2}$, $\mathrm{H}_{2} \mathrm{O}$, and $\mathrm{CO}$. It could be assumed that

$$
\sum_{i=5}^{\mathrm{m}} y_{i, \mathrm{~A}} \ll 1
$$

Therefore, Eq. (31) can be simplified and approximated to

$$
y_{\mathrm{O}_{2}, \mathrm{~L}} \cong \frac{y_{\mathrm{N}_{2}, \mathrm{~L}}}{\left(1-y_{\mathrm{O}_{2}, \mathrm{~A}}\right)} y_{\mathrm{O}_{2}, \mathrm{~A}}
$$

\subsubsection{Scheme $\mathrm{B}\left(\mathrm{O}_{2}\right.$ and $\mathrm{CO}_{2}$ Measured $)$}

In this case, $\mathrm{H}_{2} \mathrm{O}$ and $\mathrm{CO}$ are removed by the traps, and $\mathrm{O}_{2}, \mathrm{~N}_{2}, \mathrm{CO}_{2}$, and $S_{i}(i=5 \ldots m)$ are the tie components in the $\mathbf{S}$-stream and the $\mathbf{A}$-stream. Then,

$$
\begin{gathered}
n_{\mathrm{T}, \mathrm{W}}=0, \\
n_{\mathrm{T}, \mathrm{A}}=n_{\mathrm{O}_{2}, \mathrm{~A}}+n_{\mathrm{N}_{2}, \mathrm{~A}}+n_{\mathrm{CO}_{2}, \mathrm{~A}}+\sum_{i=5}^{\mathrm{m}} n_{i, \mathrm{~A}}, \\
n_{\mathrm{H}_{2} \mathrm{O}, \mathrm{A}}=n_{\mathrm{CO}, \mathrm{A}}=0, \\
y_{\mathrm{O}_{2}, \mathrm{~A}}+y_{\mathrm{N}_{2}, \mathrm{~A}}+y_{\mathrm{CO}_{2}, \mathrm{~A}}+\sum_{i=5}^{\mathrm{m}} y_{i, \mathrm{~A}}=1,
\end{gathered}
$$

and 


$$
\left[\begin{array}{c}
n_{\mathrm{O}_{2}, \mathrm{~S}} \\
n_{\mathrm{N}_{2}, \mathrm{~S}} \\
n_{\mathrm{CO}_{2}, \mathrm{~S}} \\
n_{5, \mathrm{~S}} \\
\vdots \\
n_{\mathrm{m}, \mathrm{S}}
\end{array}\right]=\left[\begin{array}{c}
n_{\mathrm{O}_{2}, \mathrm{~A}} \\
n_{\mathrm{N}_{2}, \mathrm{~A}} \\
n_{\mathrm{CO}_{2}, \mathrm{~A}} \\
n_{5, \mathrm{~A}} \\
\vdots \\
n_{\mathrm{m}, \mathrm{A}}
\end{array}\right]=k_{1}\left[\begin{array}{c}
n_{\mathrm{O}_{2}, \mathrm{~L}} \\
n_{\mathrm{N}_{2}, \mathrm{~L}} \\
n_{\mathrm{CO}_{2} \mathrm{~L}} \\
n_{5, \mathrm{~L}} \\
\vdots \\
n_{\mathrm{m}, \mathrm{L}}
\end{array}\right]
$$

Following the same procedure described in scheme A, it can be easily shown that

$$
\left[\begin{array}{c}
y_{\mathrm{O}_{2}, \mathrm{~L}} \\
y_{\mathrm{N}_{2}, \mathrm{~L}} \\
y_{\mathrm{CO}_{2}, \mathrm{~L}} \\
y_{5, \mathrm{~L}} \\
\vdots \\
y_{\mathrm{m}, \mathrm{L}}
\end{array}\right]=\left(y_{\mathrm{O}_{2}, \mathrm{~L}}+y_{\mathrm{N}_{2}, \mathrm{~L}}+y_{\mathrm{CO}_{2}, \mathrm{~L}}+\sum_{i=5}^{\mathrm{m}} y_{i, \mathrm{~L}}\right)\left[\begin{array}{c}
y_{\mathrm{O}_{2}, \mathrm{~A}} \\
y_{\mathrm{N}_{2}, \mathrm{~A}} \\
y_{\mathrm{CO}_{2, \mathrm{~A}}} \\
y_{5, \mathrm{~A}} \\
\vdots \\
y_{\mathrm{m}, \mathrm{A}}
\end{array}\right] .
$$

Using Eq. (10), Eq. (36) can be expressed as

$$
\left[\begin{array}{c}
y_{\mathrm{O}_{2}, \mathrm{~L}} \\
y_{\mathrm{N}_{2}, \mathrm{~L}} \\
y_{\mathrm{CO}_{2} \mathrm{~L}} \\
y_{5, \mathrm{~L}} \\
\vdots \\
y_{\mathrm{m}, \mathrm{L}}
\end{array}\right]=\left(1-y_{\mathrm{H}_{2} \mathrm{O}, \mathrm{L}}-y_{\mathrm{CO}, \mathrm{L}}\right)\left[\begin{array}{c}
y_{\mathrm{O}_{2}, \mathrm{~A}} \\
y_{\mathrm{N}_{2}, \mathrm{~A}} \\
y_{\mathrm{CO}_{2}, \mathrm{~A}} \\
y_{5, \mathrm{~A}} \\
\vdots \\
y_{\mathrm{m}, \mathrm{A}}
\end{array}\right]
$$

Equation (36) can be rewritten as

$$
\left[\begin{array}{ccccccc}
\left(1-y_{\mathrm{O}_{2}, \mathrm{~A}}\right) & -y_{\mathrm{O}_{2}, \mathrm{~A}} & -y_{\mathrm{O}_{2}, \mathrm{~A}} & -y_{\mathrm{O}_{2}, \mathrm{~A}} & -y_{\mathrm{O}_{2}, \mathrm{~A}} & \cdots & -y_{\mathrm{O}_{2}, \mathrm{~A}} \\
-y_{\mathrm{N}_{2}, \mathrm{~A}} & \left(1-y_{\mathrm{N}_{2}, \mathrm{~A}}\right) & -y_{\mathrm{N}_{2}, \mathrm{~A}} & -y_{\mathrm{N}_{2}, \mathrm{~A}} & -y_{\mathrm{N}_{2}, \mathrm{~A}} & \cdots & -y_{\mathrm{N}_{2}, \mathrm{~A}} \\
-y_{\mathrm{CO}_{2}, \mathrm{~A}} & -y_{\mathrm{CO}_{2}, \mathrm{~A}} & \left(1-y_{\mathrm{CO}_{2}, \mathrm{~A}}\right) & -y_{\mathrm{CO}_{2}, \mathrm{~A}} & -y_{\mathrm{CO}_{2}, \mathrm{~A}} & \cdots & -y_{\mathrm{CO}_{2}, \mathrm{~A}} \\
-y_{5, \mathrm{~A}} & -y_{5, \mathrm{~A}} & -y_{5, \mathrm{~A}} & \left(1-y_{5, \mathrm{~A}}\right) & -y_{5, \mathrm{~A}} & \cdots & -y_{5, \mathrm{~A}} \\
\vdots & \vdots & \vdots & \vdots & \vdots & \ddots & \vdots \\
-y_{\mathrm{m}, \mathrm{A}} & -y_{\mathrm{m}, \mathrm{A}} & -y_{\mathrm{m}, \mathrm{A}} & -y_{\mathrm{m}, \mathrm{A}} & -y_{\mathrm{m}, \mathrm{A}} & \cdots & \left(1-y_{\mathrm{m}, \mathrm{A}}\right)
\end{array}\right]\left[\begin{array}{c}
y_{\mathrm{O}_{2}, \mathrm{~L}} \\
y_{\mathrm{N}_{2}, \mathrm{~L}} \\
y_{\mathrm{CO}_{2}, \mathrm{~L}} \\
y_{5, \mathrm{~L}} \\
\vdots \\
y_{\mathrm{m}, \mathrm{L}}
\end{array}\right]=\left[\begin{array}{c}
0 \\
0 \\
0 \\
0 \\
\vdots \\
0
\end{array}\right] .
$$

The $(m-1) \times(m-1)$ coefficient matrix has a rank of $(m-2)$; therefore, only $(m-2)$ equations are independent [16]. The matrices with the rows associated with $y_{\mathrm{O}_{2}, \mathrm{~L}}, y_{\mathrm{CO}_{2}, \mathrm{~L}}$, and $y_{5, \mathrm{~L}} \cdots y_{\mathrm{m}, \mathrm{L}}$ are only considered. Equation (38) can be manipulated and reduced to the following form.

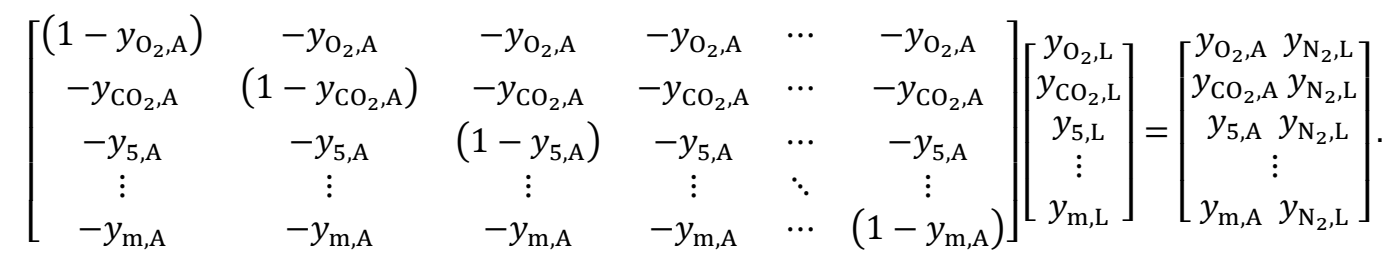

Solving for $y_{\mathrm{O}_{2}, \mathrm{~L}}, y_{\mathrm{CO}_{2}, \mathrm{~L}}$, and $y_{5, \mathrm{~L}} \cdots y_{\mathrm{m}, \mathrm{L}}$, 


$$
\left[\begin{array}{c}
y_{\mathrm{O}_{2}, \mathrm{~L}} \\
y_{\mathrm{CO}_{2}, \mathrm{~L}} \\
y_{5, \mathrm{~L}} \\
\vdots \\
y_{\mathrm{m}, \mathrm{L}}
\end{array}\right]=\left[\begin{array}{cccccc}
\left(1-y_{\mathrm{O}_{2}, \mathrm{~A}}\right) & -y_{\mathrm{O}_{2}, \mathrm{~A}} & -y_{\mathrm{O}_{2}, \mathrm{~A}} & -y_{\mathrm{O}_{2}, \mathrm{~A}} & \cdots & -y_{\mathrm{O}_{2}, \mathrm{~A}} \\
-y_{\mathrm{CO}_{2}, \mathrm{~A}} & \left(1-y_{\mathrm{CO}_{2}, \mathrm{~A}}\right) & -y_{\mathrm{CO}_{2}, \mathrm{~A}} & -y_{\mathrm{CO}_{2}, \mathrm{~A}} & \cdots & -y_{\mathrm{CO}_{2}, \mathrm{~A}} \\
-y_{5, \mathrm{~A}} & -y_{5, \mathrm{~A}} & \left(1-y_{5, \mathrm{~A}}\right) & -y_{5, \mathrm{~A}} & \cdots & -y_{5, \mathrm{~A}} \\
\vdots & \vdots & \vdots & \vdots & \ddots & \vdots \\
-y_{\mathrm{m}, \mathrm{A}} & -y_{\mathrm{m}, \mathrm{A}} & -y_{\mathrm{m}, \mathrm{A}} & -y_{\mathrm{m}, \mathrm{A}} & \cdots & \left(1-y_{\mathrm{m}, \mathrm{A}}\right)
\end{array}\right]^{-1}\left[\begin{array}{cc}
y_{\mathrm{O}_{2}, \mathrm{~A}} & y_{\mathrm{N}_{2}, \mathrm{~L}} \\
y_{\mathrm{CO}_{2}, \mathrm{~A}} y_{\mathrm{N}_{2}, \mathrm{~L}} \\
y_{5, \mathrm{~A}} & y_{\mathrm{N}_{2}, \mathrm{~L}} \\
\vdots \\
y_{\mathrm{m}, \mathrm{A}} & y_{\mathrm{N}_{2}, \mathrm{~L}}
\end{array}\right] .
$$

Simplifying by using the inverse of the above matrix given in Appendix A, Eq. (40) becomes

$$
\left[\begin{array}{c}
y_{\mathrm{O}_{2}, \mathrm{~L}} \\
y_{\mathrm{CO}_{2, \mathrm{~L}}} \\
y_{5, \mathrm{~L}} \\
\vdots \\
y_{\mathrm{m}, \mathrm{L}}
\end{array}\right]=\frac{y_{\mathrm{N}_{2}, \mathrm{~L}}}{\left(1-y_{\mathrm{O}_{2}, \mathrm{~A}}-y_{\mathrm{CO}_{2}, \mathrm{~A}}-\sum_{i=5}^{\mathrm{m}} y_{i, \mathrm{~A}}\right)}\left[\begin{array}{c}
y_{\mathrm{O}_{2}, \mathrm{~A}} \\
y_{\mathrm{CO}_{2, \mathrm{~A}}} \\
y_{5, \mathrm{~A}} \\
\vdots \\
y_{\mathrm{m}, \mathrm{A}}
\end{array}\right] .
$$

Similar to scheme A, with $\sum_{i=5}^{\mathrm{m}} y_{i, \mathrm{~A}} \ll 1$, Eq. (41) simplifies to the following equation.

$$
\left[\begin{array}{c}
y_{\mathrm{O}_{2}, \mathrm{~L}} \\
y_{\mathrm{CO}_{2}, \mathrm{~L}}
\end{array}\right] \cong \frac{y_{\mathrm{N}_{2} \mathrm{~L}}}{\left(1-y_{\mathrm{O}_{2}, \mathrm{~A}}-y_{\mathrm{CO}_{2}, \mathrm{~A}}\right)}\left[\begin{array}{c}
y_{\mathrm{O}_{2}, \mathrm{~A}} \\
y_{\mathrm{CO}_{2}, \mathrm{~A}}
\end{array}\right]
$$

\subsubsection{Scheme $\mathrm{C}\left(\mathrm{O}_{2}, \mathrm{CO}_{2}\right.$, and $\mathrm{CO}$ Measured $)$}

Under this condition, $\mathrm{H}_{2} \mathrm{O}$ is removed by the traps, and $\mathrm{O}_{2}, \mathrm{~N}_{2}, \mathrm{CO}_{2}, \mathrm{CO}$, and $S_{i}(i=5 \ldots \mathrm{m})$ are the tie components in the $\mathbf{S}$-stream and the $\mathbf{A}$-stream. Then,

$$
\begin{gathered}
n_{\mathrm{T}, \mathrm{W}}=0, \\
n_{\mathrm{T}, \mathrm{A}}=n_{\mathrm{O}_{2}, \mathrm{~A}}+n_{\mathrm{N}_{2}, \mathrm{~A}}+n_{\mathrm{CO}_{2}, \mathrm{~A}}+n_{\mathrm{CO}, \mathrm{A}}+\sum_{i=5}^{\mathrm{m}} n_{i, \mathrm{~A}}, \\
n_{\mathrm{H}_{2} \mathrm{O}, \mathrm{A}}=0, \\
y_{\mathrm{O}_{2}, \mathrm{~A}}+y_{\mathrm{N}_{2}, \mathrm{~A}}+y_{\mathrm{CO}_{2}, \mathrm{~A}}+y_{\mathrm{CO}, \mathrm{A}}+\sum_{i=5}^{\mathrm{m}} y_{i, \mathrm{~A}}=1,
\end{gathered}
$$

and

$$
\left[\begin{array}{c}
n_{\mathrm{O}_{2}, \mathrm{~S}} \\
n_{\mathrm{N}_{2}, \mathrm{~S}} \\
n_{\mathrm{CO}_{2}, \mathrm{~S}} \\
n_{\mathrm{CO}, \mathrm{S}} \\
n_{5, \mathrm{~S}} \\
\vdots \\
n_{\mathrm{m}, \mathrm{S}}
\end{array}\right]=\left[\begin{array}{c}
n_{\mathrm{O}_{2}, \mathrm{~A}} \\
n_{\mathrm{N}_{2}, \mathrm{~A}} \\
n_{\mathrm{CO}_{2}, \mathrm{~A}} \\
n_{\mathrm{CO}, \mathrm{A}} \\
n_{5, \mathrm{~A}} \\
\vdots \\
n_{\mathrm{m}, \mathrm{A}}
\end{array}\right]=k_{1}\left[\begin{array}{c}
n_{\mathrm{O}_{2}, \mathrm{~L}} \\
n_{\mathrm{N}_{2}, \mathrm{~L}} \\
n_{\mathrm{CO}_{2}, \mathrm{~L}} \\
n_{\mathrm{CO}, \mathrm{L}} \\
n_{5, \mathrm{~L}} \\
\vdots \\
n_{\mathrm{m}, \mathrm{L}}
\end{array}\right]
$$

Following the same procedure described in schemes A and B, it can be easily shown that 


$$
\left[\begin{array}{c}
y_{\mathrm{O}_{2}, \mathrm{~L}} \\
y_{\mathrm{N}_{2}, \mathrm{~L}} \\
y_{\mathrm{CO}_{2}, \mathrm{~L}} \\
y_{\mathrm{CO}, \mathrm{L}} \\
y_{5, \mathrm{~L}} \\
\vdots \\
y_{\mathrm{m}, \mathrm{L}}
\end{array}\right]=\left(y_{\mathrm{O}_{2} \mathrm{~L}}+y_{\mathrm{N}_{2} \mathrm{~L}}+y_{\mathrm{CO}_{2} \mathrm{~L}}+y_{\mathrm{CO}, \mathrm{L}}+\sum_{i=5}^{\mathrm{m}} y_{i, \mathrm{~L}}\right)\left[\begin{array}{c}
y_{\mathrm{O}_{2}, \mathrm{~A}} \\
y_{\mathrm{N}_{2}, \mathrm{~A}} \\
y_{\mathrm{CO}_{2}, \mathrm{~A}} \\
y_{\mathrm{CO}, \mathrm{A}} \\
y_{5, \mathrm{~A}} \\
\vdots \\
y_{\mathrm{m}, \mathrm{A}}
\end{array}\right]
$$

Using Eq. (10), Eq. (45) becomes

$$
\left[\begin{array}{c}
y_{\mathrm{O}_{2}, \mathrm{~L}} \\
y_{\mathrm{N}_{2}, \mathrm{~L}} \\
y_{\mathrm{CO}_{2} \mathrm{~L}} \\
y_{\mathrm{CO}, \mathrm{L}} \\
y_{5, \mathrm{~L}} \\
\vdots \\
y_{\mathrm{m}, \mathrm{L}}
\end{array}\right]=\left(1-y_{\mathrm{H}_{2} \mathrm{O}, \mathrm{L}}\right)\left[\begin{array}{c}
y_{\mathrm{O}_{2}, \mathrm{~A}} \\
y_{\mathrm{N}_{2}, \mathrm{~A}} \\
y_{\mathrm{CO}_{2}, \mathrm{~A}} \\
y_{\mathrm{CO}, \mathrm{A}} \\
y_{5, \mathrm{~A}} \\
\vdots \\
y_{\mathrm{m}, \mathrm{A}}
\end{array}\right]
$$

Equation (45) can be rewritten as

$$
\left[\begin{array}{ccccccc}
\left(1-y_{\mathrm{O}_{2}, \mathrm{~A}}\right) & -y_{\mathrm{O}_{2}, \mathrm{~A}} & -y_{\mathrm{O}_{2}, \mathrm{~A}} & -y_{\mathrm{O}_{2}, \mathrm{~A}} & -y_{\mathrm{O}_{2}, \mathrm{~A}} & \cdots & -y_{\mathrm{O}_{2}, \mathrm{~A}} \\
-y_{\mathrm{N}_{2}, \mathrm{~A}} & \left(1-y_{\mathrm{N}_{2}, \mathrm{~A}}\right) & -y_{\mathrm{N}_{2}, \mathrm{~A}} & -y_{\mathrm{N}_{2}, \mathrm{~A}} & -y_{\mathrm{N}_{2}, \mathrm{~A}} & \cdots & -y_{\mathrm{N}_{2}, \mathrm{~A}} \\
-y_{\mathrm{CO}_{2}, \mathrm{~A}} & -y_{\mathrm{CO}_{2}, \mathrm{~A}} & \left(1-y_{\mathrm{CO}_{2}, \mathrm{~A}}\right) & -y_{\mathrm{CO}_{2}, \mathrm{~A}} & -y_{\mathrm{CO}_{2}, \mathrm{~A}} & \cdots & -y_{\mathrm{CO}_{2}, \mathrm{~A}} \\
-y_{\mathrm{CO}, \mathrm{A}} & -y_{\mathrm{CO}, \mathrm{A}} & -y_{\mathrm{CO}, \mathrm{A}} & \left(1-y_{\mathrm{CO}, \mathrm{A}}\right) & -y_{\mathrm{CO}, \mathrm{A}} & \cdots & -y_{\mathrm{CO}, \mathrm{A}} \\
-y_{5, \mathrm{~A}} & -y_{5, \mathrm{~A}} & -y_{5, \mathrm{~A}} & -y_{5, \mathrm{~A}} & \left(1-y_{5, \mathrm{~A}}\right) & \cdots & -y_{5, \mathrm{~A}} \\
\vdots & \vdots & \vdots & \vdots & \vdots & \ddots & \vdots \\
-y_{\mathrm{m}, \mathrm{A}} & -y_{\mathrm{m}, \mathrm{A}} & -y_{\mathrm{m}, \mathrm{A}} & -y_{\mathrm{m}, \mathrm{A}} & -y_{\mathrm{m}, \mathrm{A}} & \cdots & \left(1-y_{\mathrm{m}, \mathrm{A}}\right)
\end{array}\right]\left[\begin{array}{c}
y_{\mathrm{O}_{2}, \mathrm{~L}} \\
y_{\mathrm{N}_{2}, \mathrm{~L}} \\
y_{\mathrm{CO}_{2}, \mathrm{~L}} \\
y_{\mathrm{CO}, \mathrm{L}} \\
y_{5, \mathrm{~L}} \\
\vdots \\
y_{\mathrm{m}, \mathrm{L}}
\end{array}\right]=\left[\begin{array}{c}
0 \\
0 \\
0 \\
0 \\
0 \\
\vdots \\
0
\end{array}\right] \cdot(47)
$$

The $(\mathrm{m} \times \mathrm{m})$ coefficient matrix has a rank of $(m-1)$; therefore, only $(m-1)$ equations are independent [16]. The equations with $y_{\mathrm{O}_{2}, \mathrm{~L}}, y_{\mathrm{CO}_{2}, \mathrm{~L}}, y_{\mathrm{CO}, \mathrm{L}}$, and $y_{5, \mathrm{~L}} \cdots y_{\mathrm{m}, \mathrm{L}}$ are only considered. Equation (47) can be manipulated and reduced to the following form.

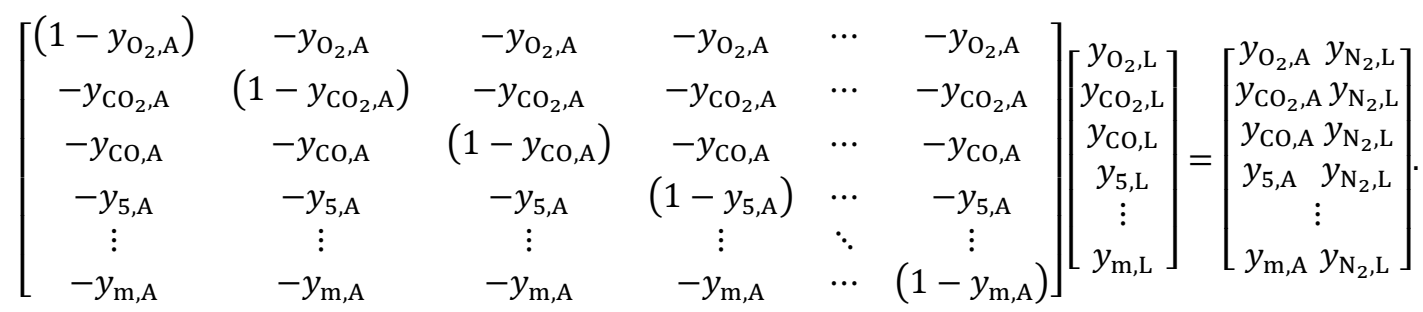

Solving for $y_{\mathrm{O}_{2}, \mathrm{~L}}, y_{\mathrm{CO}_{2}, \mathrm{~L}}, y_{\mathrm{CO}, \mathrm{L}}$, and $y_{5, \mathrm{~L}} \cdots y_{\mathrm{m}, \mathrm{L}}$,

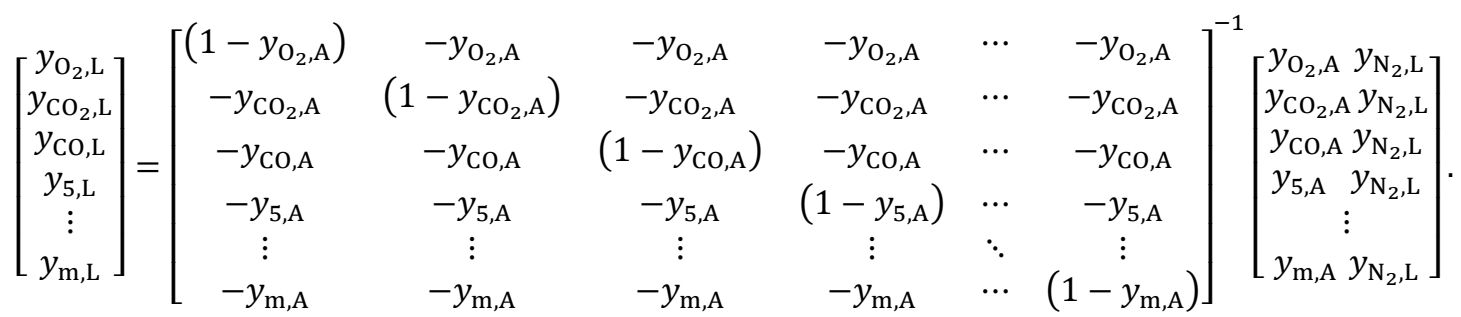


Simplifying by using the inverse of the above matrix given in Appendix A, Eq. (48) becomes

$$
\left[\begin{array}{c}
y_{\mathrm{O}_{2}, \mathrm{~L}} \\
y_{\mathrm{CO}_{2} \mathrm{~L}} \\
y_{\mathrm{CO}, \mathrm{L}} \\
y_{5, \mathrm{~L}} \\
\vdots \\
y_{\mathrm{m}, \mathrm{L}}
\end{array}\right]=\frac{y_{\mathrm{N}_{2}, \mathrm{~L}}}{\left(1-y_{\mathrm{O}_{2}, \mathrm{~A}}-y_{\mathrm{CO}_{2}, \mathrm{~A}}-y_{\mathrm{CO}, \mathrm{A}}-\sum_{i=5}^{\mathrm{m}} y_{i, \mathrm{~A}}\right)}\left[\begin{array}{c}
y_{\mathrm{O}_{2}, \mathrm{~A}} \\
y_{\mathrm{CO}_{2}, \mathrm{~A}} \\
y_{\mathrm{CO}, \mathrm{A}} \\
y_{5, \mathrm{~A}} \\
\vdots \\
y_{\mathrm{m}, \mathrm{A}}
\end{array}\right] .
$$

Similar to schemes $\mathrm{A}$ and $\mathrm{B}$, with $\sum_{i=5}^{\mathrm{m}} y_{i, \mathrm{~A}} \ll 1$, Eq. (49) simplifies to the following equation.

$$
\left[\begin{array}{c}
y_{\mathrm{O}_{2}, \mathrm{~L}} \\
y_{\mathrm{CO}_{2}, \mathrm{~L}} \\
y_{\mathrm{CO}, \mathrm{L}}
\end{array}\right] \cong \frac{y_{\mathrm{N}_{2}, \mathrm{~L}}}{\left(1-y_{\mathrm{O}_{2}, \mathrm{~A}}-y_{\mathrm{CO}_{2}, \mathrm{~A}}-y_{\mathrm{CO}, \mathrm{A}}\right)}\left[\begin{array}{c}
y_{\mathrm{O}_{2}, \mathrm{~A}} \\
y_{\mathrm{CO}_{2, \mathrm{~A}}} \\
y_{\mathrm{CO}, \mathrm{A}}
\end{array}\right]
$$

\subsubsection{Scheme D $\left(\mathrm{O}_{2}, \mathrm{CO}_{2}, \mathrm{CO}\right.$, and $\mathrm{H}_{2} \mathrm{O}$ Measured $)$}

In this case, $\mathrm{H}_{2} \mathrm{O}$ is still removed by the traps, and $\mathrm{O}_{2}, \mathrm{~N}_{2}, \mathrm{CO}_{2}, \mathrm{CO}$, and $S_{i}(i=5 \ldots \mathrm{m})$ are the tie components in the $\mathbf{S}$-stream and the $\mathbf{A}$-stream, which is identical to scheme C. However, one has

and

$$
n_{\mathrm{T}, \mathrm{W}}=k_{2} n_{\mathrm{T}, \mathrm{L}}
$$

$$
y_{\mathrm{H}_{2} \mathrm{O}, \mathrm{L}}=y_{\mathrm{H}_{2} \mathrm{O}, \mathrm{W}}
$$

Equation (46) with $y_{\mathrm{H}_{2} \mathrm{O}, \mathrm{L}}=y_{\mathrm{H}_{2} \mathrm{O}, \mathrm{W}}$ and Eq. (50) are equally applicable to scheme D.

\subsubsection{Remarks on Schemes A, B, C, and D}

In the above four measurement schemes $\mathrm{A}, \mathrm{B}, \mathrm{C}$, and $\mathrm{D}$, the equations that relate $y_{\mathrm{O}_{2}, \mathrm{~L}}$ to $y_{\mathrm{O}_{2}, \mathrm{~A}}$ require knowing $y_{\mathrm{N}_{2}, \mathrm{~L}}$. If there is no nitrogen generation during burning, then $n_{\mathrm{N}_{2}, \mathrm{~L}}=n_{\mathrm{N}_{2}, \mathrm{E}}$. Hence,

$$
y_{\mathrm{N}_{2}, \mathrm{~L}}=\frac{n_{\mathrm{T}, \mathrm{E}}}{n_{\mathrm{T}, \mathrm{L}}} y_{\mathrm{N}_{2}, \mathrm{E}}
$$

Even if there is generation of nitrogen during burning, due to the copious amount of entrained air into the collection hood, $n_{\mathrm{N}_{2}, \mathrm{E}} \gg n_{\mathrm{N}_{2}, \mathrm{rxn}}$; therefore, $n_{\mathrm{N}_{2}, \mathrm{~L}}=n_{\mathrm{N}_{2}, \mathrm{E}}+n_{\mathrm{N}_{2}, \mathrm{rxn}} \cong n_{\mathrm{N}_{2}, \mathrm{E}}$. Equation (52) could still be approximately valid. Equation (52) was used by Parker [6, 7] and others [8-11].

If other major combustion product species are to be included in the dry-basis measurements, a generalized scheme where $j(<m)$ major combustion species and $\mathrm{O}_{2}$ are measured can also be easily derived based on the generalized framework, and it is provided in Appendix $\mathrm{C}$ for reference.

\subsection{Measurements of the E-Stream}

The species concentrations in the $\mathbf{E}$-stream can be obtained from background measurements before the initiation of a fire test. An implicit assumption is that the background concentrations of $\mathrm{O}_{2}, \mathrm{~N}_{2}, \mathrm{CO}_{2}$, and $\mathrm{H}_{2} \mathrm{O}$ do not change during the entire course of a fire test. When no fire is present (denoted the symbols with superscript $*$ ), no combustion products are generated, and the following conditions are applied to schemes $\mathrm{A}^{*}, \mathrm{~B}^{*}, \mathrm{C}^{*}$, and $\mathrm{D}^{*}$. 


$$
\begin{gathered}
n_{\mathrm{T}, \mathrm{E}}^{*}=n_{\mathrm{T}, \mathrm{L}}^{*} \\
{\left[\begin{array}{c}
n_{\mathrm{O}_{2}, \mathrm{E}} \\
n_{\mathrm{N}_{2}, \mathrm{E}} \\
n_{\mathrm{CO}_{2}, \mathrm{E}} \\
n_{\mathrm{H}_{2} \mathrm{O}, \mathrm{E}}
\end{array}\right] \equiv\left[\begin{array}{c}
n_{\mathrm{O}_{2}, \mathrm{E}}^{*} \\
n_{\mathrm{N}_{2}, \mathrm{E}}^{*} \\
n_{\mathrm{CO}_{2}, \mathrm{E}}^{*} \\
n_{\mathrm{H}_{2} \mathrm{O}, \mathrm{E}}^{*}
\end{array}\right]=\left[\begin{array}{c}
n_{\mathrm{O}_{2}, \mathrm{~L}}^{*} \\
n_{\mathrm{N}_{2}, \mathrm{~L}}^{*} \\
n_{\mathrm{CO}_{2}, \mathrm{~L}}^{*} \\
n_{\mathrm{H}_{2} \mathrm{O}, \mathrm{L}}^{*}
\end{array}\right] .} \\
{\left[\begin{array}{c}
y_{\mathrm{O}_{2}, \mathrm{E}} \\
y_{\mathrm{N}_{2}, \mathrm{E}} \\
y_{\mathrm{CO}_{2}, \mathrm{E}} \\
y_{\mathrm{H}_{2} \mathrm{O}, \mathrm{E}}
\end{array}\right] \equiv\left[\begin{array}{c}
y_{\mathrm{O}_{2}, \mathrm{E}}^{*} \\
y_{\mathrm{N}_{2}, \mathrm{E}}^{*} \\
y_{\mathrm{CO}_{2}, \mathrm{E}}^{*} \\
y_{\mathrm{H}_{2} \mathrm{O}, \mathrm{E}}^{*}
\end{array}\right]=\left[\begin{array}{c}
y_{\mathrm{O}_{2}, \mathrm{~L}}^{*} \\
y_{\mathrm{N}_{2}, \mathrm{~L}}^{*} \\
y_{\mathrm{CO}}^{*}, \mathrm{~L} \\
y_{\mathrm{H}_{2} \mathrm{O}, \mathrm{L}}^{*}
\end{array}\right] .} \\
y_{\mathrm{O}_{2}, \mathrm{E}}^{*}+y_{\mathrm{N}_{2}, \mathrm{E}}^{*}+y_{\mathrm{CO}, \mathrm{E}}^{*}+y_{\mathrm{H}_{2} \mathrm{O}, \mathrm{E}}^{*}=1 . \\
y_{\mathrm{CO}, \mathrm{E}}^{*}=y_{\mathrm{CO}, \mathrm{L}}^{*}=y_{\mathrm{CO}, \mathrm{S}}^{*}=y_{\mathrm{CO}, \mathrm{A}}^{*}=0 . \\
y_{i, \mathrm{~L}}^{*}=y_{i, \mathrm{~S}}^{*}=y_{i, \mathrm{~A}}^{*}=0 \quad i=5, \cdots \mathrm{m} . \\
\beta^{*}=0 .
\end{gathered}
$$

\subsubsection{Scheme $A^{*}$ (No Fire, $\mathrm{O}_{2}$ Measured)}

In this case, $\mathrm{H}_{2} \mathrm{O}$ and $\mathrm{CO}_{2}$ are removed by the traps, and $\mathrm{O}_{2}$ and $\mathrm{N}_{2}$ are the tie components in the $\mathbf{S}$ stream and the $\mathbf{A}$-stream. Then,

$$
\begin{gathered}
n_{\mathrm{T}, \mathrm{W}}^{*}=0, \\
n_{\mathrm{T}, \mathrm{A}}^{*}=n_{\mathrm{O}_{2}, \mathrm{~A}}^{*}+n_{\mathrm{N}_{2}, \mathrm{~A}}^{*}, \\
n_{\mathrm{H}_{2} \mathrm{O}, \mathrm{A}}^{*}=n_{\mathrm{CO}_{2}, \mathrm{~A}}^{*}=n_{\mathrm{CO}, \mathrm{A}}^{*}=0, \\
y_{\mathrm{O}_{2}, \mathrm{~A}}^{*}+y_{\mathrm{N}_{2}, \mathrm{~A}}^{*}=1,
\end{gathered}
$$

and

$$
\left[\begin{array}{l}
n_{\mathrm{O}_{2}, \mathrm{~S}}^{*} \\
n_{\mathrm{N}_{2}, \mathrm{~S}}^{*}
\end{array}\right]=\left[\begin{array}{c}
n_{\mathrm{O}_{2}, \mathrm{~A}}^{*} \\
n_{\mathrm{N}_{2}, \mathrm{~A}}^{*}
\end{array}\right]=k_{1}\left[\begin{array}{c}
n_{\mathrm{O}_{2}, \mathrm{~L}}^{*} \\
n_{\mathrm{N}_{2}, \mathrm{~L}}^{*}
\end{array}\right]
$$

Under no-fire condition, it can be easily shown by the same procedure described in scheme A that Eq. (33) is exact and is not an approximation.

$$
y_{\mathrm{O}_{2}, \mathrm{~L}}^{*}=\frac{y_{\mathrm{N}_{2}, \mathrm{~L}}^{*}}{\left(1-y_{\mathrm{O}_{2}, \mathrm{~A}}^{*}\right)} y_{\mathrm{O}_{2}, \mathrm{~A}}^{*}
$$

From Eq. (55), one has 


$$
y_{\mathrm{O}_{2}, \mathrm{E}}^{*}=\frac{y_{\mathrm{N}_{2}, \mathrm{E}}^{*}}{\left(1-y_{\mathrm{O}_{2}, \mathrm{~A}}^{*}\right)} y_{\mathrm{O}_{2}, \mathrm{~A}}^{*}
$$

Substituting Eq. (56) into Eq. (65) and simplifying, one obtains

$$
y_{\mathrm{O}_{2}, \mathrm{E}}^{*}=\frac{\left(1-y_{\mathrm{O}_{2}, \mathrm{E}}^{*}-y_{\mathrm{CO}_{2}, \mathrm{E}}^{*}-y_{\mathrm{H}_{2} \mathrm{O}, \mathrm{E}}^{*}\right)}{\left(1-y_{\mathrm{O}_{2}, \mathrm{~A}}^{*}\right)} y_{\mathrm{O}_{2}, \mathrm{~A}}^{*},
$$

and

$$
y_{\mathrm{O}_{2}, \mathrm{E}}^{*}=\left(1-y_{\mathrm{CO}_{2}, \mathrm{E}}^{*}-y_{\mathrm{H}_{2} \mathrm{O}, \mathrm{E}}^{*}\right) y_{\mathrm{O}_{2}, \mathrm{~A}}^{*}
$$

Independent measurements of $y_{\mathrm{CO}_{2}, \mathrm{E}}^{*}$ and $y_{\mathrm{H}_{2} \mathrm{O}, \mathrm{E}}^{*}$ are required to obtain $y_{\mathrm{O}_{2}, \mathrm{E}}^{*}$ if $\mathrm{CO}_{2}$ and $\mathrm{H}_{2} \mathrm{O}$ are removed by the traps. If the relative humidity $(R H)$ in the $\mathbf{E}$-stream is measured, then

$$
y_{\mathrm{H}_{2} \mathrm{O}, \mathrm{E}}^{*}=\frac{R H}{100} \frac{p_{\mathrm{vap}, \mathrm{H}_{2} \mathrm{O}}\left(T_{\mathrm{E}}\right)}{p_{\mathrm{T}}},
$$

where $p_{\mathrm{vap}, \mathrm{H}_{2} \mathrm{O}}\left(T_{\mathrm{E}}\right)$ is the vapor pressure of water at temperature $T_{\mathrm{E}}$, and $p_{\mathrm{T}}$ is the ambient total pressure.

\subsubsection{Scheme $\mathrm{B}^{*}$ (No Fire, $\mathrm{O}_{2}$ and $\mathrm{CO}_{2}$ Measured)}

In this case, only $\mathrm{H}_{2} \mathrm{O}$ is removed by the traps, since no $\mathrm{CO}$ is generated, and $\mathrm{O}_{2}, \mathrm{CO}_{2}$, and $\mathrm{N}_{2}$ are the tie components in the $\mathbf{S}$-stream and the $\mathbf{A}$-stream. Then,

$$
\begin{gathered}
n_{\mathrm{T}, \mathrm{W}}^{*}=0, \\
n_{\mathrm{T}, \mathrm{A}}^{*}=n_{\mathrm{O}_{2}, \mathrm{~A}}^{*}+n_{\mathrm{N}_{2}, \mathrm{~A}}^{*}+n_{\mathrm{CO}_{2}, \mathrm{~A}}^{*}, \\
n_{\mathrm{H}_{2} \mathrm{O}, \mathrm{A}}^{*}=n_{\mathrm{CO}, \mathrm{A}}^{*}=0, \\
y_{\mathrm{O}_{2}, \mathrm{~A}}^{*}+y_{\mathrm{N}_{2}, \mathrm{~A}}^{*}+y_{\mathrm{CO}_{2}, \mathrm{~A}}^{*}=1,
\end{gathered}
$$

and

$$
\left[\begin{array}{c}
n_{\mathrm{O}_{2}, \mathrm{~S}}^{*} \\
n_{\mathrm{N}_{2}, \mathrm{~S}}^{*} \\
n_{\mathrm{CO}_{2}, \mathrm{~S}}^{*}
\end{array}\right]=\left[\begin{array}{c}
n_{\mathrm{O}_{2}, \mathrm{~A}}^{*} \\
n_{\mathrm{N}_{2}, \mathrm{~A}}^{*} \\
n_{\mathrm{CO}_{2}, \mathrm{~A}}^{*}
\end{array}\right]=k_{1}\left[\begin{array}{c}
n_{\mathrm{O}_{2}, \mathrm{~L}}^{*} \\
n_{\mathrm{N}_{2}, \mathrm{~L}}^{*} \\
n_{\mathrm{CO}_{2}, \mathrm{~L}}^{*}
\end{array}\right] .
$$

Under the no-fire condition, it can be easily shown by the same procedure described in scheme B that Eq. (42) is exact and is not an approximation.

$$
\left[\begin{array}{c}
y_{\mathrm{O}_{2}, \mathrm{~L}}^{*} \\
y_{\mathrm{CO}_{2}, \mathrm{~L}}^{*}
\end{array}\right]=\frac{y_{\mathrm{N}_{2}, \mathrm{~L}}^{*}}{\left(1-y_{\mathrm{O}_{2}, \mathrm{~A}}^{*}-y_{\mathrm{CO}_{2}, \mathrm{~A}}^{*}\right)}\left[\begin{array}{c}
y_{\mathrm{O}_{2}, \mathrm{~A}}^{*} \\
y_{\mathrm{CO}_{2}, \mathrm{~A}}^{*}
\end{array}\right]
$$

From Eq. (55), one has 


$$
\left[\begin{array}{c}
y_{\mathrm{O}_{2}, \mathrm{E}}^{*} \\
y_{\mathrm{CO}_{2}, \mathrm{E}}^{*}
\end{array}\right]=\frac{y_{\mathrm{N}_{2}, \mathrm{E}}^{*}}{\left(1-y_{\mathrm{O}_{2}, \mathrm{~A}}^{*}-y_{\mathrm{CO}_{2}, \mathrm{~A}}^{*}\right)}\left[\begin{array}{c}
y_{\mathrm{O}_{2}, \mathrm{~A}}^{*} \\
y_{\mathrm{CO}_{2}, \mathrm{~A}}^{*}
\end{array}\right] .
$$

Substituting $y_{\mathrm{N}_{2}, \mathrm{E}}^{*}=\left(1-y_{\mathrm{O}_{2}, \mathrm{E}}^{*}-y_{\mathrm{CO}_{2}, \mathrm{E}}^{*}-y_{\mathrm{H}_{2} \mathrm{O}, \mathrm{E}}^{*}\right)$ into Eq. (71) and solving for $y_{\mathrm{O}_{2}, \mathrm{E}}^{*}$ and $y_{\mathrm{CO}_{2}, \mathrm{E}}^{*}$, one obtains

$$
\left[\begin{array}{c}
y_{\mathrm{O}_{2}, \mathrm{E}}^{*} \\
y_{\mathrm{CO}_{2}, \mathrm{E}}^{*}
\end{array}\right]=\frac{\left(1-y_{\mathrm{O}_{2}, \mathrm{E}}^{*}-y_{\mathrm{CO}_{2}, \mathrm{E}}^{*}-y_{\mathrm{H}_{2} \mathrm{O}, \mathrm{E}}^{*}\right)}{\left(1-y_{\mathrm{O}_{2}, \mathrm{~A}}^{*}-y_{\mathrm{CO}_{2}, \mathrm{~A}}^{*}\right)}\left[\begin{array}{c}
y_{\mathrm{O}_{2}, \mathrm{~A}}^{*} \\
y_{\mathrm{CO}_{2}, \mathrm{~A}}^{*}
\end{array}\right]
$$

and

$$
\left[\begin{array}{c}
y_{\mathrm{O}_{2}, \mathrm{E}}^{*} \\
y_{\mathrm{CO}_{2}, \mathrm{E}}^{*}
\end{array}\right]=\left(1-y_{\mathrm{H}_{2} \mathrm{O}, \mathrm{E}}^{*}\right)\left[\begin{array}{c}
y_{\mathrm{O}_{2}, \mathrm{~A}}^{*} \\
y_{\mathrm{CO}_{2}, \mathrm{~A}}^{*}
\end{array}\right] .
$$

Since $\mathrm{H}_{2} \mathrm{O}$ is removed by the traps, independent measurement of $y_{\mathrm{H}_{2} \mathrm{O}, \mathrm{E}}^{*}$ is required to obtain $y_{\mathrm{O}_{2}, \mathrm{E}}^{*}$ using Eq. (67) if the relative humidity $(R H)$ in the E-stream is measured.

With $y_{\mathrm{H}_{2} \mathrm{O}, \mathrm{E}}^{*}$ obtained and $y_{\mathrm{O}_{2}, \mathrm{E}}^{*}$ and $y_{\mathrm{CO}_{2}, \mathrm{E}}^{*}$ from Eq. (72), then $y_{\mathrm{N}_{2}, \mathrm{E}}^{*}$ can be calculated using Eq. (56).

$$
y_{\mathrm{N}_{2}, \mathrm{E}}^{*}=\left(1-y_{\mathrm{O}_{2}, \mathrm{E}}^{*}-y_{\mathrm{CO}_{2}, \mathrm{E}}^{*}-y_{\mathrm{H}_{2} \mathrm{O}, \mathrm{E}}^{*}\right) .
$$

\subsubsection{Scheme $\mathrm{C}^{*}$ (No Fire, $\mathrm{O}_{2}, \mathrm{CO}_{2}$, and $\mathrm{CO}$ Measured)}

Under this condition, $\mathrm{H}_{2} \mathrm{O}$ is removed by the traps, and $\mathrm{O}_{2}, \mathrm{~N}_{2}$, and $\mathrm{CO}_{2}$ are the tie components in the $\mathbf{S}$-stream and the $\mathbf{A}$-stream, since there is no fire, and no $\mathrm{CO}$ is produced. Therefore, scheme $\mathrm{C}^{*}$ (no fire) is identical to scheme $\mathrm{B}^{*}$ (no fire) described above. All the equations derived in scheme $\mathrm{B}^{*}$ (no fire) are equally applicable to scheme $C^{*}$ (no fire).

\subsubsection{Scheme D* (No Fire, $\mathrm{O}_{2}, \mathrm{CO}_{2}, \mathrm{CO}$, and $\mathrm{H}_{2} \mathrm{O}$ Measured)}

In this case, $\mathrm{H}_{2} \mathrm{O}$ is still removed by the traps, and $\mathrm{O}_{2}, \mathrm{~N}_{2}$, and $\mathrm{CO}_{2}$ are the tie components in the $\mathbf{S}-$ stream and the A-stream. Similar to scheme $C^{*}$ (no fire), scheme $D^{*}$ (no fire) is identical to scheme $B^{*}$ (no fire). All the equations derived in scheme $\mathrm{B}^{*}$ (no fire) above are equally applicable to scheme $\mathrm{D}^{*}$ (no fire). The following two additional equations also apply to scheme $\mathrm{D}^{*}$ (no fire).

$$
\begin{gathered}
n_{\mathrm{T}, \mathrm{W}}^{*}=k_{2} n_{\mathrm{T}, \mathrm{L}}^{*} \neq 0 . \\
y_{\mathrm{H}_{2} \mathrm{O}, \mathrm{E}}^{*}=y_{\mathrm{H}_{2} \mathrm{O}, \mathrm{L}}^{*}=y_{\mathrm{H}_{2} \mathrm{O}, \mathrm{W} .}^{*} .
\end{gathered}
$$

With $y_{\mathrm{H}_{2} \mathrm{O}, \mathrm{E}}^{*}$ known, Eq. (72) can be used to obtain $y_{\mathrm{O}_{2}, \mathrm{E}}^{*}$.

\section{Oxygen Depletion Factor}

The expressions for the oxygen depletion factor $\phi$ used for heat release rate calculations for the four gas measurement schemes can now be obtained using Eq. (3). 


\subsection{Scheme A}

Substituting Eq. (33) and Eq. (52) into Eq. (3),

$$
\phi=1-\frac{n_{\mathrm{T}, \mathrm{L}} y_{\mathrm{O}_{2}, \mathrm{~L}}}{n_{\mathrm{T}, \mathrm{E}} y_{\mathrm{O}_{2}, \mathrm{E}}}=1-\frac{y_{\mathrm{N}_{2}, \mathrm{E}}}{y_{\mathrm{O}_{2}, \mathrm{E}}} \frac{y_{\mathrm{O}_{2}, \mathrm{~A}}}{\left(1-y_{\mathrm{O}_{2}, \mathrm{~A}}\right)} .
$$

Substituting Eq. (65) into Eq. (74), simplifying, and noting that $y_{\mathrm{O}_{2}, \mathrm{E}}^{*} \equiv y_{\mathrm{O}_{2}, \mathrm{E}}$ and $y_{\mathrm{N}_{2}, \mathrm{E}}^{*} \equiv y_{\mathrm{N}_{2}, \mathrm{E}}$, one obtains

$$
\phi=\frac{y_{\mathrm{O}_{2}, \mathrm{~A}}^{*}-y_{\mathrm{O}_{2}, \mathrm{~A}}}{y_{\mathrm{O}_{2}, \mathrm{~A}}^{*}\left(1-y_{\mathrm{O}_{2}, \mathrm{~A}}\right)} .
$$

\subsection{Scheme B}

Substituting Eq. (42) and Eq. (52) into Eq. (3),

$$
\phi=1-\frac{n_{\mathrm{T}, \mathrm{L}} y_{\mathrm{O}_{2}, \mathrm{~L}}}{n_{\mathrm{T}, \mathrm{E}} y_{\mathrm{O}_{2}, \mathrm{E}}}=1-\frac{y_{\mathrm{N}_{2}, \mathrm{E}}}{y_{\mathrm{O}_{2}, \mathrm{E}}} \frac{y_{\mathrm{O}_{2}, \mathrm{~A}}}{\left(1-y_{\mathrm{O}_{2}, \mathrm{~A}}-y_{\mathrm{CO}_{2}, \mathrm{~A}}\right)} .
$$

Substituting Eq. (71) into Eq. (76), simplifying, and noting that $y_{\mathrm{O}_{2}, \mathrm{E}}^{*} \equiv y_{\mathrm{O}_{2}, \mathrm{E}}$ and $y_{\mathrm{N}_{2}, \mathrm{E}}^{*} \equiv y_{\mathrm{N}_{2}, \mathrm{E}}$, one obtains

$$
\phi=1-\frac{y_{\mathrm{O}_{2}, \mathrm{~A}}\left(1-y_{\mathrm{O}_{2}, \mathrm{~A}}^{*}-y_{\mathrm{CO}_{2}, \mathrm{~A}}^{*}\right)}{y_{\mathrm{O}_{2}, \mathrm{~A}}^{*}\left(1-y_{\mathrm{O}_{2}, \mathrm{~A}}-y_{\mathrm{CO}_{2}, \mathrm{~A}}\right)^{\prime}}
$$

and

$$
\phi=\frac{y_{\mathrm{O}_{2}, \mathrm{~A}}^{*}\left(1-y_{\mathrm{CO}_{2}, \mathrm{~A}}\right)-y_{\mathrm{O}_{2}, \mathrm{~A}}\left(1-y_{\mathrm{CO}_{2}, \mathrm{~A}}^{*}\right)}{y_{\mathrm{O}_{2}, \mathrm{~A}}^{*}\left(1-y_{\mathrm{O}_{2}, \mathrm{~A}}-y_{\mathrm{CO}_{2}, \mathrm{~A}}\right)}
$$

\subsection{Scheme C}

Substituting Eq. (50) and Eq. (52) into Eq. (3),

$$
\phi=1-\frac{n_{\mathrm{T}, \mathrm{L}} y_{\mathrm{O}_{2}, \mathrm{~L}}}{n_{\mathrm{T}, \mathrm{E}} y_{\mathrm{O}_{2}, \mathrm{E}}}=1-\frac{y_{\mathrm{N}_{2}, \mathrm{E}}}{y_{\mathrm{O}_{2}, \mathrm{E}}} \frac{y_{\mathrm{O}_{2}, \mathrm{~A}}}{\left(1-y_{\mathrm{O}_{2}, \mathrm{~A}}-y_{\mathrm{CO}_{2}, \mathrm{~A}}-y_{\mathrm{CO}, \mathrm{A}}\right)}
$$

Substituting Eq. (71) into Eq. (78), simplifying, and noting that $y_{\mathrm{O}_{2}, \mathrm{E}}^{*} \equiv y_{\mathrm{O}_{2}, \mathrm{E}}$ and $y_{\mathrm{N}_{2}, \mathrm{E}}^{*} \equiv y_{\mathrm{N}_{2}, \mathrm{E}}$, one obtains

$$
\phi=1-\frac{y_{\mathrm{O}_{2}, \mathrm{~A}}\left(1-y_{\mathrm{O}_{2}, \mathrm{~A}}^{*}-y_{\mathrm{CO}_{2}, \mathrm{~A}}^{*}\right)}{y_{\mathrm{O}_{2}, \mathrm{~A}}^{*}\left(1-y_{\mathrm{O}_{2}, \mathrm{~A}}-y_{\mathrm{CO}_{2}, \mathrm{~A}}-y_{\mathrm{CO}, \mathrm{A}}\right)^{\prime}}
$$

and 


$$
\phi=\frac{y_{\mathrm{O}_{2}, \mathrm{~A}}^{*}\left(1-y_{\mathrm{CO}_{2}, \mathrm{~A}}-y_{\mathrm{CO}, \mathrm{A}}\right)-y_{\mathrm{O}_{2}, \mathrm{~A}}\left(1-y_{\mathrm{CO}_{2}, \mathrm{~A}}^{*}\right)}{y_{\mathrm{O}_{2}, \mathrm{~A}}^{*}\left(1-y_{\mathrm{O}_{2}, \mathrm{~A}}-y_{\mathrm{CO}_{2}, \mathrm{~A}}-y_{\mathrm{CO}, \mathrm{A}}\right)} .
$$

\subsection{Scheme D}

Since the $\mathrm{O}_{2}, \mathrm{CO}_{2}$, and $\mathrm{CO}$ measurement schemes are similar to scheme C, Eq. (78) is equally applied to scheme D.

\subsection{Remarks on Oxygen Depletion Factor}

It should be noted that the oxygen depletion factor $\phi$ for scheme $\mathrm{A}$ and cheme $\mathrm{B}$ can be obtained from $\phi$ for scheme C, Eq. (79), simply by making $y_{\mathrm{CO}_{2}, \mathrm{~A}}=y_{\mathrm{CO}, \mathrm{A}}=y_{\mathrm{CO}_{2}, \mathrm{~A}}^{*}=0$ and $y_{\mathrm{CO}, \mathrm{A}}=0$,respectively. A generalized oxygen depletion factor for the generalized measurement scheme where $j(<m)$ major combustion product species are to be included in the dry-basis measurements is provided in Appendix $\mathrm{C}$ for reference.

\section{Determination of $\boldsymbol{n}_{\mathrm{T}, \mathrm{L}}$ or $\boldsymbol{n}_{\mathrm{T}, \mathrm{E}}$}

The total material flow rate in the $\mathbf{L}$-stream is given by

$$
n_{\mathrm{T}, \mathrm{L}}=\rho_{\mathrm{L}}\left\langle v_{\mathrm{L}}\right\rangle A_{\mathrm{L}}
$$

where $\rho_{\mathrm{L}}=\sum_{i=1}^{\mathrm{m}} y_{i, \mathrm{~L}} \rho_{i}$ is the gas mixture molar density at $T_{\mathrm{L}},\left\langle v_{\mathrm{L}}\right\rangle$ is the mean velocity of the gas mixture flowing through the duct, and $A_{\mathrm{L}}$ is the cross-sectional area of the duct. Knowing $y_{i, \mathrm{~L}}$ and $\rho_{i}, \rho_{\mathrm{L}}$ can be calculated. In most of the applications, $\rho_{\mathrm{L}} \cong \rho_{\text {air }}\left(T_{\mathrm{L}}\right)$. The average gas velocity $\left\langle v_{\mathrm{L}}\right\rangle$ in the exhaust duct can be measured using bidirectional probes or annubar flow meters [4].

Another technique that can be used to measure $n_{\mathrm{T}, \mathrm{E}}$ and $n_{\mathrm{T}, \mathrm{L}}$ is the tracer gas method, where a tracer gas is injected steadily at a known flow rate into the exhaust duct far enough upstream to ensure complete mixing with the exhaust stream before it reaches the gas sampling point for subsequent measurements of its concentration.

Let the material flow rate of tracer gas injected into the exhaust duct, $n_{\mathrm{tr}, \mathrm{L}}$, be known. Then, the total flow rate in the exhaust duct now becomes

$$
\hat{n}_{\mathrm{T}, \mathrm{L}}=n_{\mathrm{T}, \mathrm{L}}+n_{\mathrm{tr}, \mathrm{L}}
$$

Since $n_{\mathrm{T}, \mathrm{L}} \gg n_{\mathrm{tr}, \mathrm{L}}$, one can approximate $\hat{n}_{\mathrm{T}, \mathrm{L}} \cong n_{\mathrm{T}, \mathrm{L}}$. If the tracer gas concentration can be measured in the $\mathbf{W}$-stream directly and knowing that $y_{\mathrm{tr}, \mathrm{L}}=y_{\mathrm{tr}, \mathrm{W}}$, then

$$
n_{\mathrm{T}, \mathrm{L}}=\frac{n_{\mathrm{tr}, \mathrm{L}}}{y_{\mathrm{tr}, \mathrm{L}}}=\frac{n_{\mathrm{tr}, \mathrm{L}}}{y_{\mathrm{tr}, \mathrm{W}}}
$$

If the tracer gas concentration is measured in the A-stream, one of the above four measurement schemes used to obtain $y_{\mathrm{tr}, \mathrm{L}}$ will now by discussed.

\subsection{Scheme A}

It can be easily shown that Eq. (31) can be modified to accommodate the tracer gas as follows. 


$$
\left[\begin{array}{c}
y_{\mathrm{O}_{2}, \mathrm{~L}} \\
y_{\mathrm{tr}, \mathrm{L}} \\
y_{5, \mathrm{~L}} \\
\vdots \\
y_{\mathrm{m}, \mathrm{L}}
\end{array}\right]=\frac{y_{\mathrm{N}_{2}, \mathrm{~L}}}{\left(1-y_{\mathrm{O}_{2}, \mathrm{~A}}-y_{\mathrm{tr}, \mathrm{A}}-\sum_{i=5}^{\mathrm{m}} y_{i, \mathrm{~A}}\right)}\left[\begin{array}{c}
y_{\mathrm{O}_{2}, \mathrm{~A}} \\
y_{\mathrm{tr}, \mathrm{A}} \\
y_{5, \mathrm{~A}} \\
\vdots \\
y_{\mathrm{m}, \mathrm{A}}
\end{array}\right]
$$

It could again be assumed that

$$
\sum_{i=5}^{\mathrm{m}} y_{i, \mathrm{~A}} \ll 1
$$

Then, Eq. (82) can be simplified and approximated to

$$
\left[\begin{array}{c}
y_{\mathrm{O}_{2}, \mathrm{~L}} \\
y_{\mathrm{tr}, \mathrm{L}}
\end{array}\right]=\frac{y_{\mathrm{N}_{2}, \mathrm{~L}}}{\left(1-y_{\mathrm{O}_{2}, \mathrm{~A}}-y_{\mathrm{tr}, \mathrm{A}}\right)}\left[\begin{array}{c}
y_{\mathrm{O}_{2}, \mathrm{~A}} \\
y_{\mathrm{tr}, \mathrm{A}}
\end{array}\right]
$$

Substituting Eq. (52) into Eq. (83),

$$
\left[\begin{array}{c}
y_{\mathrm{O}_{2}, \mathrm{~L}} \\
y_{\mathrm{tr}, \mathrm{L}}
\end{array}\right]=\frac{\mathrm{n}_{\mathrm{T}, \mathrm{E}}}{n_{\mathrm{T}, \mathrm{L}}} \frac{y_{\mathrm{N}_{2}, \mathrm{E}}}{\left(1-y_{\mathrm{O}_{2}, \mathrm{~A}}-y_{\mathrm{tr}, \mathrm{A}}\right)}\left[\begin{array}{c}
y_{\mathrm{O}_{2}, \mathrm{~A}} \\
y_{\mathrm{tr}, \mathrm{A}}
\end{array}\right]
$$

From Eq. (84),

$$
y_{\mathrm{tr}, \mathrm{L}} n_{\mathrm{T}, \mathrm{L}}=\frac{y_{\mathrm{N}_{2}, \mathrm{E}} y_{\mathrm{tr}, \mathrm{A}}}{\left(1-y_{\mathrm{O}_{2}, \mathrm{~A}}-y_{\mathrm{tr}, \mathrm{A}}\right)} n_{\mathrm{T}, \mathrm{E}}
$$

With $y_{\mathrm{N}_{2}, \mathrm{E}} \equiv y_{\mathrm{N}_{2}, \mathrm{E}}^{*}$ and $n_{\mathrm{tr}, \mathrm{L}}=y_{\mathrm{tr}, \mathrm{L}} n_{\mathrm{T}, \mathrm{L}}$,

$$
n_{\mathrm{T}, \mathrm{E}}=\frac{n_{\mathrm{tr}, \mathrm{L}}\left(1-y_{\mathrm{O}_{2}, \mathrm{~A}}-y_{\mathrm{tr}, \mathrm{A}}\right)}{y_{\mathrm{N}_{2}, \mathrm{E}}^{*} y_{\mathrm{tr}, \mathrm{A}}}
$$

\subsection{Scheme B}

Similar to scheme A, it can be easily shown that Eq. (41) can be modified to accommodate the tracer gas as follows.

$$
\left[\begin{array}{c}
y_{\mathrm{O}_{2}, \mathrm{~L}} \\
y_{\mathrm{CO}_{2}, \mathrm{~L}} \\
y_{\mathrm{tr}, \mathrm{L}} \\
y_{5, \mathrm{~L}} \\
\vdots \\
y_{\mathrm{m}, \mathrm{L}}
\end{array}\right]=\frac{y_{\mathrm{N}_{2}, \mathrm{~L}}}{\left(1-y_{\mathrm{O}_{2}, \mathrm{~A}}-y_{\mathrm{CO}_{2}, \mathrm{~A}}-y_{\mathrm{tr}, \mathrm{A}}-\sum_{i=5}^{\mathrm{m}} y_{i, \mathrm{~A}}\right)}\left[\begin{array}{c}
y_{\mathrm{O}_{2}, \mathrm{~A}} \\
y_{\mathrm{CO}_{2}, \mathrm{~A}} \\
y_{\mathrm{tr}, \mathrm{A}} \\
y_{5, \mathrm{~A}} \\
\vdots \\
y_{\mathrm{m}, \mathrm{A}}
\end{array}\right]
$$

Again, with $\sum_{i=5}^{\mathrm{m}} y_{i, \mathrm{~A}} \ll 1$, Eq. (86) simplifies to the following equation.

$$
\left[\begin{array}{c}
y_{\mathrm{O}_{2}, \mathrm{~L}} \\
y_{\mathrm{CO}_{2}, \mathrm{~L}} \\
y_{\mathrm{tr}, \mathrm{L}}
\end{array}\right]=\frac{y_{\mathrm{N}_{2}, \mathrm{~L}}}{\left(1-y_{\mathrm{O}_{2}, \mathrm{~A}}-y_{\mathrm{CO}_{2}, \mathrm{~A}}-y_{\mathrm{tr}, \mathrm{A}}\right)}\left[\begin{array}{c}
y_{\mathrm{O}_{2}, \mathrm{~A}} \\
y_{\mathrm{CO}_{2}, \mathrm{~A}} \\
y_{\mathrm{tr}, \mathrm{A}}
\end{array}\right]
$$


Substituting Eq. (52) into Eq. (87),

$$
\left[\begin{array}{c}
y_{\mathrm{O}_{2}, \mathrm{~L}} \\
y_{\mathrm{CO}_{2}, \mathrm{~L}} \\
y_{\mathrm{tr}, \mathrm{L}}
\end{array}\right]=\frac{n_{\mathrm{T}, \mathrm{E}}}{n_{\mathrm{T}, \mathrm{L}}} \frac{y_{\mathrm{N}_{2}, \mathrm{E}}}{\left(1-y_{\mathrm{O}_{2}, \mathrm{~A}}-y_{\mathrm{CO}_{2}, \mathrm{~A}}-y_{\mathrm{tr}, \mathrm{A}}\right)}\left[\begin{array}{c}
y_{\mathrm{O}_{2}, \mathrm{~A}} \\
y_{\mathrm{CO}_{2}, \mathrm{~A}} \\
y_{\mathrm{tr}, \mathrm{A}}
\end{array}\right]
$$

From Eq. (88),

$$
y_{\mathrm{tr}, \mathrm{L}} n_{\mathrm{T}, \mathrm{L}}=\frac{y_{\mathrm{N}_{2}, \mathrm{E}} y_{\mathrm{tr}, \mathrm{A}}}{\left(1-y_{\mathrm{O}_{2}, \mathrm{~A}}-y_{\mathrm{CO}_{2}, \mathrm{~A}}-y_{\mathrm{tr}, \mathrm{A}}\right)} n_{\mathrm{T}, \mathrm{E}}
$$

With $y_{\mathrm{N}_{2}, \mathrm{E}} \equiv y_{\mathrm{N}_{2}, \mathrm{E}}^{*}$ and $n_{\mathrm{tr}, \mathrm{L}}=y_{\mathrm{tr}, \mathrm{L}} n_{\mathrm{T}, \mathrm{L}}$,

$$
n_{\mathrm{T}, \mathrm{E}}=\frac{n_{\mathrm{tr}, \mathrm{L}}\left(1-y_{\mathrm{O}_{2}, \mathrm{~A}}-y_{\mathrm{CO}_{2}, \mathrm{~A}}-y_{\mathrm{tr}, \mathrm{A}}\right)}{y_{\mathrm{N}_{2}, \mathrm{E}}^{*} y_{\mathrm{tr}, \mathrm{A}}}
$$

\subsection{Scheme C}

Similar to schemes A and B, it can be easily shown that Eq. (49) can be modified to accommodate the tracer gas as follows.

$$
\left[\begin{array}{c}
y_{\mathrm{O}_{2}, \mathrm{~L}} \\
y_{\mathrm{CO}_{2}, \mathrm{~L}} \\
y_{\mathrm{CO}, \mathrm{L}} \\
y_{\mathrm{tr}, \mathrm{L}} \\
y_{5, \mathrm{~L}} \\
\vdots \\
y_{\mathrm{m}, \mathrm{L}}
\end{array}\right]=\frac{y_{\mathrm{N}_{2}, \mathrm{~L}}}{\left(1-y_{\mathrm{O}_{2}, \mathrm{~A}}-y_{\mathrm{CO}_{2}, \mathrm{~A}}-y_{\mathrm{CO}, \mathrm{A}}-y_{\mathrm{tr}, \mathrm{A}}-\sum_{i=5}^{\mathrm{m}} y_{i, \mathrm{~A}}\right)}\left[\begin{array}{c}
y_{\mathrm{O}_{2}, \mathrm{~A}} \\
y_{\mathrm{CO}_{2}, \mathrm{~A}} \\
y_{\mathrm{CO}, \mathrm{A}} \\
y_{\mathrm{tr}, \mathrm{A}} \\
y_{5, \mathrm{~A}} \\
\vdots \\
y_{\mathrm{m}, \mathrm{A}}
\end{array}\right]
$$

Again, with $\sum_{i=5}^{\mathrm{m}} y_{i, \mathrm{~A}} \ll 1$, Eq. (90) simplifies to the following equation.

$$
\left[\begin{array}{c}
y_{\mathrm{O}_{2}, \mathrm{~L}} \\
y_{\mathrm{CO}_{2}, \mathrm{~L}} \\
y_{\mathrm{CO}, \mathrm{L}} \\
y_{\mathrm{tr}, \mathrm{L}}
\end{array}\right]=\frac{y_{\mathrm{N}_{2}, \mathrm{~L}}}{\left(1-y_{\mathrm{O}_{2}, \mathrm{~A}}-y_{\mathrm{CO}_{2}, \mathrm{~A}}-y_{\mathrm{CO}, \mathrm{A}}-y_{\mathrm{tr}, \mathrm{A}}\right)}\left[\begin{array}{c}
y_{\mathrm{O}_{2}, \mathrm{~A}} \\
y_{\mathrm{CO}_{2}, \mathrm{~A}} \\
y_{\mathrm{CO}, \mathrm{A}} \\
y_{\mathrm{tr}, \mathrm{A}}
\end{array}\right]
$$

Substituting Eq. (52) into Eq. (91),

$$
\left[\begin{array}{c}
y_{\mathrm{O}_{2}, \mathrm{~L}} \\
y_{\mathrm{CO}_{2}, \mathrm{~L}} \\
y_{\mathrm{CO}, \mathrm{L}} \\
y_{\mathrm{tr}, \mathrm{L}}
\end{array}\right]=\frac{n_{\mathrm{T}, \mathrm{E}}}{n_{\mathrm{T}, \mathrm{L}}} \frac{y_{\mathrm{N}_{2}, \mathrm{E}}}{\left(1-y_{\mathrm{O}_{2}, \mathrm{~A}}-y_{\mathrm{CO}_{2}, \mathrm{~A}}-y_{\mathrm{CO}, \mathrm{A}}-y_{\mathrm{tr}, \mathrm{A}}\right)}\left[\begin{array}{c}
y_{\mathrm{O}_{2}, \mathrm{~A}} \\
y_{\mathrm{CO}_{2}, \mathrm{~A}} \\
y_{\mathrm{CO}, \mathrm{A}} \\
y_{\mathrm{tr}, \mathrm{A}}
\end{array}\right] .
$$

From Eq. (92),

$$
y_{\mathrm{tr}, \mathrm{L}} n_{\mathrm{T}, \mathrm{L}}=\frac{y_{\mathrm{N}_{2}, \mathrm{E}} y_{\mathrm{tr}, \mathrm{A}}}{\left(1-y_{\mathrm{O}_{2}, \mathrm{~A}}-y_{\mathrm{CO}_{2}, \mathrm{~A}}-y_{\mathrm{CO}, \mathrm{A}}-y_{\mathrm{tr}, \mathrm{A}}\right)} n_{\mathrm{T}, \mathrm{E}}
$$

With $y_{\mathrm{N}_{2}, \mathrm{E}} \equiv y_{\mathrm{N}_{2}, \mathrm{E}}^{*}$ and $n_{\mathrm{tr}, \mathrm{L}}=y_{\mathrm{tr}, \mathrm{L}} n_{\mathrm{T}, \mathrm{L}}$, 


$$
n_{\mathrm{T}, \mathrm{E}}=\frac{n_{\mathrm{tr}, \mathrm{L}}\left(1-y_{\mathrm{O}_{2}, \mathrm{~A}}-y_{\mathrm{CO}_{2}, \mathrm{~A}}-y_{\mathrm{CO}, \mathrm{A}}-y_{\mathrm{tr}, \mathrm{A}}\right)}{y_{\mathrm{N}_{2}, \mathrm{E}}^{*} y_{\mathrm{tr}, \mathrm{A}}} .
$$

\subsection{Scheme D}

Since the $\mathrm{O}_{2}, \mathrm{CO}_{2}$, and $\mathrm{CO}$ measurement schemes are similar to scheme C, Eq. (93) for scheme $\mathrm{C}$ is equally applicable to scheme $\mathrm{D}$.

\section{Summary}

This paper revisits the derivations of formulas commonly used in heat release rate measurements based on the oxygen consumption principle. The derivations are aided by treating the oxygen consumption calorimeter operation as a chemical engineering process with a representative flow diagram. If the oxygen consumption calorimeter is treated as a chemical flow process and analyzed as such, the analysis is intuitive and straightforward. The derivations have been presented pedagogically so that they can be easily followed, while at the same time maintaining rigor of analysis and generalization with few assumptions. The three parameters essential to the calculations of heat release rates of burning materials in fire tests are $\phi, y_{\mathrm{O}_{2}, \mathrm{E}}$, and $n_{\mathrm{T}, \mathrm{E}}$ or $n_{\mathrm{T}, \mathrm{L}}$ as related in Eq. (4). Table 1 and Table 2 summarize, respectively, the appropriate formulas for $\phi$ and $y_{\mathrm{O}_{2}, \mathrm{E}}$ that can be used to obtain heat release rates based on the four gas measurement schemes commonly used in fire tests. The parameter $n_{\mathrm{T}, \mathrm{L}}$ can be measured using either annubar or bidirectional probes, or the tracer-gas technique can be used to measure $n_{\mathrm{T}, \mathrm{E}}$ or $n_{\mathrm{T}, \mathrm{L}}$. It is hoped that the inclusion of the detailed derivation procedures will allow practitioners new to the field to better comprehend the governing physics and assumptions used in heat release rate calculations. The developed framework is generalized, and so it can be extended to include other combustion product species and different suites of gas measurement schemes. This paper could also serve as supplementary material to Parker's NBS report [6].

Table 1. Summary of formulas for $\phi$.

\begin{tabular}{|c|c|c|}
\hline Scheme & Formula & $\begin{array}{l}\text { Equation } \\
\text { (this paper) }\end{array}$ \\
\hline Scheme A $\left(\mathrm{O}_{2}\right.$ measured $)$ & $\phi=\frac{y_{\mathrm{O}_{2}, \mathrm{~A}}^{*}-y_{\mathrm{O}_{2}, \mathrm{~A}}}{y_{\mathrm{O}_{2}, \mathrm{~A}}^{*}\left(1-y_{\mathrm{O}_{2}, \mathrm{~A}}\right)}$ & Eq. (75) \\
\hline Scheme $\mathrm{B}\left(\mathrm{O}_{2}\right.$ and $\mathrm{CO}_{2}$ measured $)$ & $\phi=\frac{y_{\mathrm{O}_{2}, \mathrm{~A}}^{*}\left(1-y_{\mathrm{CO}_{2}, \mathrm{~A}}\right)-y_{\mathrm{O}_{2}, \mathrm{~A}}\left(1-y_{\mathrm{CO}_{2}, \mathrm{~A}}^{*}\right)}{y_{\mathrm{O}_{2}, \mathrm{~A}}^{*}\left(1-y_{\mathrm{O}_{2}, \mathrm{~A}}-y_{\mathrm{CO}_{2}, \mathrm{~A}}\right)}$ & Eq. (77) \\
\hline $\begin{array}{l}\text { Scheme } \mathrm{C}\left(\mathrm{O}_{2}, \mathrm{CO}_{2} \text {, and } \mathrm{CO}\right. \\
\text { measured })\end{array}$ & $\phi=\frac{y_{\mathrm{O}_{2}, \mathrm{~A}}^{*}\left(1-y_{\mathrm{CO}_{2}, \mathrm{~A}}-y_{\mathrm{CO}_{2, \mathrm{~A}}}\right)-y_{\mathrm{O}_{2, \mathrm{~A}}}\left(1-y_{\mathrm{CO}_{2}, \mathrm{~A}}^{*}\right)}{y_{\mathrm{O}_{2}, \mathrm{~A}}^{*}\left(1-y_{\mathrm{O}_{2}, \mathrm{~A}}-y_{\mathrm{CO}_{2, \mathrm{~A}}}-y_{\mathrm{CO}, \mathrm{A}}\right)}$ & Eq. (79) \\
\hline $\begin{array}{l}\text { Scheme } \mathrm{D}\left(\mathrm{O}_{2}, \mathrm{CO}_{2}, \mathrm{CO} \text {, and } \mathrm{H}_{2} \mathrm{O}\right. \\
\text { measured })\end{array}$ & $\phi=\frac{y_{\mathrm{O}_{2}, \mathrm{~A}}^{*}\left(1-y_{\mathrm{CO}_{2}, \mathrm{~A}}-y_{\mathrm{CO}_{2} \mathrm{~A}}\right)-y_{\mathrm{O}_{2}, \mathrm{~A}}\left(1-y_{\mathrm{CO}_{2}, \mathrm{~A}}^{*}\right)}{y_{\mathrm{O}_{2}, \mathrm{~A}}^{*}\left(1-y_{\mathrm{O}_{2}, \mathrm{~A}}-y_{\mathrm{CO}_{2}, \mathrm{~A}}-y_{\mathrm{CO}, \mathrm{A}}\right)}$ & Eq. (79) \\
\hline
\end{tabular}


Table 2. Summary of formulas for $y_{\mathrm{O}_{2}, \mathrm{E}}$.

\begin{tabular}{|c|c|c|}
\hline Scheme & Formula & $\begin{array}{c}\text { Equation } \\
\text { (this paper) }\end{array}$ \\
\hline Scheme $A^{*}$ (no fire, $\mathrm{O}_{2}$ measured) & $\begin{array}{c}y_{\mathrm{O}_{2}, \mathrm{E}} \equiv y_{\mathrm{O}_{2}, \mathrm{E}}^{*}=\left(1-y_{\mathrm{CO}_{2}, \mathrm{E}}^{*}-y_{\mathrm{H}_{2} \mathrm{O}, \mathrm{E}}^{*}\right) y_{\mathrm{O}_{2}, \mathrm{~A}}^{*} \\
y_{\mathrm{H}_{2} \mathrm{O}, \mathrm{E}}^{*}=\frac{R H}{100} \frac{p_{\mathrm{vap}, \mathrm{H}_{2} \mathrm{O}}\left(T_{\mathrm{E}}\right)}{p_{\mathrm{T}}} \\
y_{\mathrm{CO}_{2}, \mathrm{E}}^{*} \text { measured independently }\end{array}$ & Eq. (66) \\
\hline Scheme $\mathrm{B}^{*}$ (no fire, $\mathrm{O}_{2}$ and $\mathrm{CO}_{2}$ measured) & $\begin{array}{c}y_{\mathrm{O}_{2}, \mathrm{E}} \equiv y_{\mathrm{O}_{2}, \mathrm{E}}^{*}=\left(1-y_{\mathrm{H}_{2} \mathrm{O}, \mathrm{E}}^{*}\right) y_{\mathrm{O}_{2}, \mathrm{~A}}^{*} \\
y_{\mathrm{H}_{2} \mathrm{O}, \mathrm{E}}^{*}=\frac{R H}{100} \frac{p_{\mathrm{vap}, \mathrm{H}_{2} \mathrm{O}}\left(T_{\mathrm{E}}\right)}{p_{\mathrm{T}}}\end{array}$ & Eq. (67) \\
\hline $\begin{array}{c}\text { Scheme } \mathrm{C}^{*} \text { (no fire, } \mathrm{O}_{2}, \mathrm{CO}_{2} \text {, and } \mathrm{CO} \text { measured) } \\
\text { Scheme } \mathrm{D}^{*} \text { (no fire, } \mathrm{O}_{2}, \mathrm{CO}_{2}, \mathrm{CO} \text {, and } \mathrm{H}_{2} \mathrm{O} \text { measured) }\end{array}$ & $\begin{array}{l}\text { Same as scheme B* } \\
\text { Same as scheme B* }\end{array}$ & \\
\hline
\end{tabular}

\section{Appendix A}

The inverse of the matrix in Eq. (30) is

$$
\begin{aligned}
& {\left[\begin{array}{ccccc}
\left(1-y_{0_{2, \mathrm{~A}}}\right) & -y_{0_{2, \mathrm{~A}}} & \cdots & -y_{0_{2, \mathrm{~A}}} & -y_{0_{2, \mathrm{~A}}} \\
-y_{5, \mathrm{~A}} & \left(1-y_{5, \mathrm{~A}}\right) & \cdots & -y_{5, \mathrm{~A}} & -y_{5, \mathrm{~A}} \\
-y_{6, \mathrm{~A}} & -y_{6, \mathrm{~A}} & \left(1-y_{6, \mathrm{~A}}\right) & \cdots & -y_{6, \mathrm{~A}} \\
\vdots & \vdots & \ddots & \vdots & \vdots \\
-y_{\mathrm{m}, \mathrm{A}} & -y_{\mathrm{m}, \mathrm{A}} & \cdots & -y_{\mathrm{m}, \mathrm{A}} & \left(1-y_{\mathrm{m}, \mathrm{A}}\right)
\end{array}\right]^{-1}}
\end{aligned}
$$

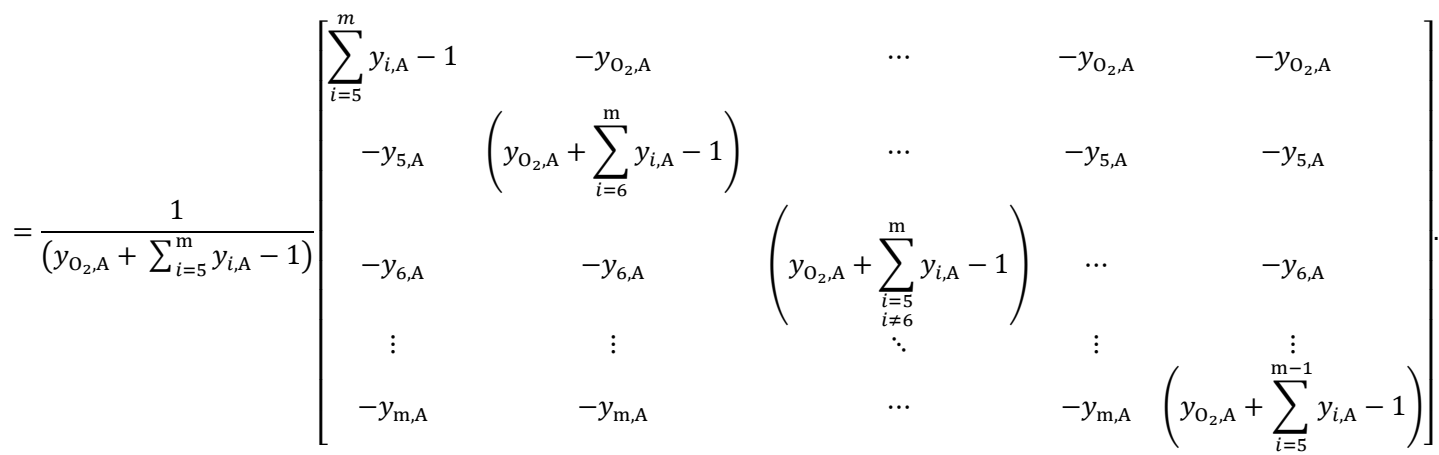

The inverse of the matrix in Eq. (40) is

$$
\left[\begin{array}{cccccc}
\left(1-y_{\mathrm{O}_{2}, \mathrm{~A}}\right) & -y_{\mathrm{O}_{2}, \mathrm{~A}} & -y_{\mathrm{O}_{2}, \mathrm{~A}} & \cdots & -y_{\mathrm{O}_{2}, \mathrm{~A}} & -y_{\mathrm{O}_{2}, \mathrm{~A}} \\
-y_{\mathrm{CO}_{2}, \mathrm{~A}} & \left(1-y_{\mathrm{CO}_{2}, \mathrm{~A}}\right) & -y_{\mathrm{CO}_{2}, \mathrm{~A}} & \cdots & -y_{\mathrm{CO}_{2}, \mathrm{~A}} & -y_{\mathrm{CO}_{2}, \mathrm{~A}} \\
-y_{5, \mathrm{~A}} & -y_{5, \mathrm{~A}} & \left(1-y_{5, \mathrm{~A}}\right) & \cdots & -y_{5, \mathrm{~A}} & -y_{5, \mathrm{~A}} \\
\vdots & \vdots & \vdots & \ddots & \ddots & \vdots \\
-y_{\mathrm{m}, \mathrm{A}} & -y_{\mathrm{m}, \mathrm{A}} & -y_{\mathrm{m}, \mathrm{A}} & \cdots & -y_{\mathrm{m}, \mathrm{A}} & \left(1-y_{\mathrm{m}, \mathrm{A}}\right)
\end{array}\right]^{-1}=\frac{\mathbf{R}}{\left(y_{\mathrm{O}_{2}, \mathrm{~A}}+y_{\mathrm{CO}_{2}, \mathrm{~A}}+\sum_{i=5}^{\mathrm{m}} y_{i, \mathrm{~A}}-1\right)^{\prime}}
$$

where $\mathbf{R}$ is equal to 


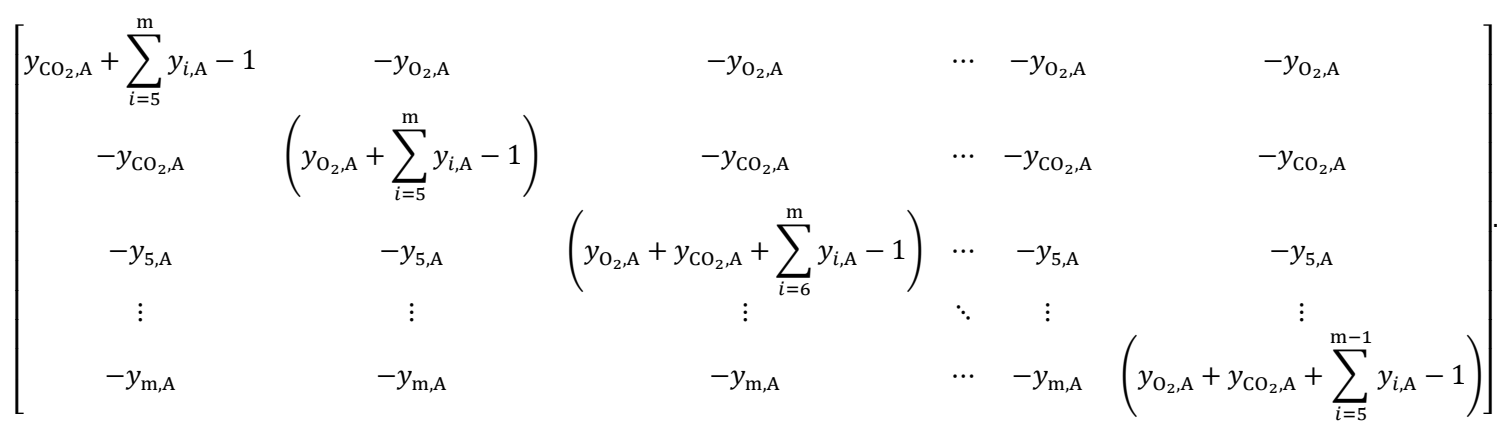

The inverse of the matrix in Eq. (48) is

$$
\left[\begin{array}{cccccc}
\left(1-y_{\mathrm{O}_{2}, \mathrm{~A}}\right) & -y_{\mathrm{O}_{2}, \mathrm{~A}} & -y_{\mathrm{O}_{2}, \mathrm{~A}} & -y_{\mathrm{O}_{2}, \mathrm{~A}} & \cdots & -y_{\mathrm{O}_{2}, \mathrm{~A}} \\
-y_{\mathrm{CO}_{2}, \mathrm{~A}} & \left(1-y_{\mathrm{CO}_{2}, \mathrm{~A}}\right) & -y_{\mathrm{CO}_{2}, \mathrm{~A}} & -y_{\mathrm{CO}_{2}, \mathrm{~A}} & \cdots & -y_{\mathrm{CO}_{2}, \mathrm{~A}} \\
-y_{\mathrm{CO}, \mathrm{A}} & -y_{\mathrm{CO}, \mathrm{A}} & \left(1-y_{\mathrm{CO}, \mathrm{A}}\right) & -y_{\mathrm{CO}, \mathrm{A}} & \cdots & -y_{\mathrm{CO}, \mathrm{A}} \\
-y_{5, \mathrm{~A}} & -y_{5, \mathrm{~A}} & -y_{5, \mathrm{~A}} & \left(1-y_{5, \mathrm{~A}}\right) & \cdots & -y_{5, \mathrm{~A}} \\
\vdots & \vdots & \vdots & \vdots & \ddots & \vdots \\
-y_{\mathrm{m}, \mathrm{A}} & -y_{\mathrm{m}, \mathrm{A}} & -y_{\mathrm{m}, \mathrm{A}} & -y_{\mathrm{m}, \mathrm{A}} & \cdots & \left(1-y_{\mathrm{m}, \mathrm{A}}\right)
\end{array}\right]^{-1}=\frac{\mathbf{Z}}{\left(y_{\mathrm{O}_{2, \mathrm{~A}}}+y_{\mathrm{CO}_{2, \mathrm{~A}}}+y_{\mathrm{CO}, \mathrm{A}}+\sum_{i=5}^{\mathrm{m}} y_{i, \mathrm{~A}}-1\right)^{\prime}}
$$

where

$$
\mathbf{Z}=\left[\begin{array}{cccccc}
z_{11} & -y_{\mathrm{O}_{2}, \mathrm{~A}} & -y_{\mathrm{O}_{2}, \mathrm{~A}} & -y_{\mathrm{O}_{2}, \mathrm{~A}} & \cdots & -y_{\mathrm{O}_{2}, \mathrm{~A}} \\
-y_{\mathrm{CO}_{2}, \mathrm{~A}} & z_{22} & -y_{\mathrm{CO}_{2}, \mathrm{~A}} & -y_{\mathrm{CO}_{2}, \mathrm{~A}} & \cdots & -y_{\mathrm{CO}_{2}, \mathrm{~A}} \\
-y_{\mathrm{CO}, \mathrm{A}} & -y_{\mathrm{CO}, \mathrm{A}} & z_{33} & -y_{\mathrm{CO}, \mathrm{A}} & \cdots & -y_{\mathrm{CO}, \mathrm{A}} \\
-y_{5, \mathrm{~A}} & -y_{5, \mathrm{~A}} & -y_{5, \mathrm{~A}} & z_{44} & \cdots & -y_{5, \mathrm{~A}} \\
\vdots & \vdots & \vdots & \vdots & \ddots & \vdots \\
-y_{\mathrm{m}, \mathrm{A}} & -y_{\mathrm{m}, \mathrm{A}} & -y_{\mathrm{m}, \mathrm{A}} & -y_{\mathrm{m}, \mathrm{A}} & \cdots & z_{(\mathrm{m}-1)(\mathrm{m}-1)}
\end{array}\right]
$$

with the diagonal elements

$$
\begin{aligned}
& z_{11}=\left(y_{\mathrm{CO}_{2}, \mathrm{~A}}+y_{\mathrm{CO}, \mathrm{A}}+\sum_{i=5}^{\mathrm{m}} y_{i, \mathrm{~A}}-1\right) \\
& z_{22}=\left(y_{\mathrm{O}_{2}, \mathrm{~A}}+y_{\mathrm{CO}, \mathrm{A}}+\sum_{i=5}^{\mathrm{m}} y_{i, \mathrm{~A}}-1\right) \\
& z_{33}=\left(y_{\mathrm{O}_{2}, \mathrm{~A}}+y_{\mathrm{CO}_{2}, \mathrm{~A}}+\sum_{i=5}^{\mathrm{m}} y_{i, \mathrm{~A}}-1\right) \\
& z_{44}=\left(y_{\mathrm{O}_{2}, \mathrm{~A}}+y_{\mathrm{CO}_{2}, \mathrm{~A}}+y_{\mathrm{CO}, \mathrm{A}}+\sum_{i=6}^{m} y_{i, \mathrm{~A}}-1\right) \\
& z_{55}=\left(y_{\mathrm{O}_{2}, \mathrm{~A}}+y_{\mathrm{CO}_{2}, \mathrm{~A}}+y_{\mathrm{CO}, \mathrm{A}}+\sum_{\substack{i=5 \\
i \neq 6}}^{\mathrm{m}} y_{i, \mathrm{~A}}-1\right)
\end{aligned}
$$




$$
\begin{aligned}
& z_{66}=\left(y_{\mathrm{O}_{2}, \mathrm{~A}}+y_{\mathrm{CO}_{2}, \mathrm{~A}}+y_{\mathrm{CO}, \mathrm{A}}+\sum_{\substack{i=5 \\
i \neq 7}}^{\mathrm{m}} y_{i, \mathrm{~A}}-1\right), \\
& \vdots \\
& z_{(\mathrm{m}-1)(\mathrm{m}-1)}=\left(y_{\mathrm{O}_{2}, \mathrm{~A}}+y_{\mathrm{CO}_{2}, \mathrm{~A}}+y_{\mathrm{CO}, \mathrm{A}}+\sum_{i=5}^{\mathrm{m}-1} y_{i, \mathrm{~A}}-1\right) .
\end{aligned}
$$

\section{Appendix B}

If the measurement scheme $\mathrm{D}$ is used to provide an independent measurement of water vapor in the $\mathbf{L}$ stream, and the measurement can be performed without removing certain combustion products from the $\mathbf{W}$ stream, $\beta$ can be determined as follows. From Eq. (12) with $n_{\mathrm{O}_{2}, \text { rxn }}=n_{\mathrm{O}_{2}, \mathrm{E}} \phi$,

$$
\beta=\left(\frac{n_{\mathrm{N}_{2}, \mathrm{~L}}-n_{\mathrm{N}_{2}, \mathrm{E}}}{n_{\mathrm{O}_{2}, \mathrm{E}} \phi}+\frac{n_{\mathrm{H}_{2} \mathrm{O}, \mathrm{L}}-n_{\mathrm{H}_{2} \mathrm{O}, \mathrm{E}}}{n_{\mathrm{O}_{2}, \mathrm{E}} \phi}+\frac{n_{\mathrm{CO}_{2}, \mathrm{~L}}-n_{\mathrm{CO}_{2}, \mathrm{E}}}{n_{\mathrm{O}_{2}, \mathrm{E}} \phi}+\frac{n_{\mathrm{CO}, \mathrm{L}}}{n_{\mathrm{O}_{2}, \mathrm{E}} \phi}+\sum_{i=5}^{\mathrm{m}} \frac{n_{i, \mathrm{~L}}}{n_{\mathrm{O}_{2}, \mathrm{E}} \phi}\right) .
$$

Equation (B1) can be rewritten as

$$
\beta y_{\mathrm{O}_{2}, \mathrm{E}} \phi n_{\mathrm{T}, \mathrm{E}}=\left(n_{\mathrm{N}_{2}, \mathrm{~L}}+n_{\mathrm{H}_{2} \mathrm{O}, \mathrm{L}}+n_{\mathrm{CO}_{2}, \mathrm{~L}}+n_{\mathrm{CO}, \mathrm{L}}+\sum_{i=5}^{\mathrm{m}} n_{i, \mathrm{~L}}\right)-\left(n_{\mathrm{N}_{2}, \mathrm{E}}+n_{\mathrm{H}_{2} \mathrm{O}, \mathrm{E}}+n_{\mathrm{CO}_{2}, \mathrm{E}}\right) .
$$

Substituting Eq. (6) and Eq. (9) into Eq. (B2),

$$
\beta y_{\mathrm{O}_{2}, \mathrm{E}} \phi n_{\mathrm{T}, \mathrm{E}}=\left(n_{\mathrm{T}, \mathrm{L}}-n_{\mathrm{O}_{2}, \mathrm{~L}}\right)-\left(n_{\mathrm{T}, \mathrm{E}}-n_{\mathrm{O}_{2}, \mathrm{E}}\right),
$$

and

$$
\beta y_{\mathrm{O}_{2}, \mathrm{E}} \phi=\left(1-y_{\mathrm{O}_{2}, \mathrm{~L}}\right) \frac{n_{\mathrm{T}, \mathrm{L}}}{n_{\mathrm{T}, \mathrm{E}}}-\left(1-y_{\mathrm{O}_{2}, \mathrm{E}}\right) .
$$

Substituting Eq. (14) into Eq. (B3) and simplifying,

$$
\beta y_{\mathrm{O}_{2}, \mathrm{E}} \phi=\left(1-y_{\mathrm{O}_{2}, \mathrm{~L}}\right)\left[1+(\beta-1) \phi y_{\mathrm{O}_{2}, \mathrm{E}}\right]-\left(1-y_{\mathrm{O}_{2}, \mathrm{E}}\right) \text {, }
$$

and

$$
\beta=\frac{(1-\phi)}{\phi} \frac{1}{y_{\mathrm{O}_{2}, \mathrm{~L}}}-\frac{1}{\phi y_{\mathrm{O}_{2}, \mathrm{E}}}+1
$$

Substituting Eq. (46) into Eq. (B4),

$$
\beta=\frac{(1-\phi)}{\phi} \frac{1}{y_{\mathrm{O}_{2}, \mathrm{~A}}\left(1-y_{\mathrm{H}_{2} \mathrm{O}, \mathrm{L}}\right)}-\frac{1}{\phi y_{\mathrm{O}_{2}, \mathrm{E}}}+1 .
$$

Knowing that $y_{\mathrm{H}_{2} \mathrm{O}, \mathrm{L}}=y_{\mathrm{H}_{2} \mathrm{O}, \mathrm{W}}$ and $y_{\mathrm{O}_{2}, \mathrm{E}} \equiv y_{\mathrm{O}_{2}, \mathrm{E}}^{*}$ and substituting Eq. (72) into Eq. (B5), 


$$
\beta=\frac{(1-\phi)}{\phi} \frac{1}{y_{\mathrm{O}_{2}, \mathrm{~A}}\left(1-y_{\mathrm{H}_{2} \mathrm{O}, \mathrm{W}}\right)}-\frac{1}{\phi y_{\mathrm{O}_{2}, \mathrm{~A}}^{*}\left(1-y_{\mathrm{H}_{2} \mathrm{O}, \mathrm{E}}^{*}\right)}+1
$$

From Eq. (73),

$$
\beta=\frac{(1-\phi)}{\phi} \frac{1}{y_{\mathrm{O}_{2}, \mathrm{~A}}\left(1-y_{\mathrm{H}_{2} \mathrm{O}, \mathrm{W}}\right)}-\frac{1}{\phi y_{\mathrm{O}_{2}, \mathrm{~A}}^{*}\left(1-y_{\mathrm{H}_{2} \mathrm{O}, \mathrm{W}}^{*}\right)}+1
$$

\section{Appendix C}

Based on the generalized framework developed here, the formulas for the four specific measurement schemes (schemes A, B, C, and D) can be easily extended to a generalized measurement scheme that involves the dry-basis measurements of $\mathrm{O}_{2}, \mathrm{CO}_{2}, \mathrm{CO}$, and $\mathrm{S}_{5}$ to $\mathrm{S}_{j}$ major combustion product species with $j<m$. With $\sum_{i=5}^{\mathrm{m}} y_{i, \mathrm{~A}}=\sum_{i=5}^{\mathrm{j}} y_{i, \mathrm{~A}}+\sum_{i=\mathrm{j}+1}^{\mathrm{m}} y_{i, \mathrm{~A}}$, one rewrites Eq. (49) as

$$
\left[\begin{array}{c}
y_{\mathrm{O}_{2}, \mathrm{~L}} \\
y_{\mathrm{CO}_{2}, \mathrm{~L}} \\
y_{\mathrm{CO}, \mathrm{L}} \\
y_{5, \mathrm{~L}} \\
\vdots \\
y_{\mathrm{m}, \mathrm{L}}
\end{array}\right]=\frac{y_{\mathrm{N}_{2} \mathrm{~L}}}{\left(1-y_{\mathrm{O}_{2}, \mathrm{~A}}-y_{\mathrm{CO}_{2}, \mathrm{~A}}-y_{\mathrm{CO}, \mathrm{A}}-\sum_{i=5}^{\mathrm{j}} y_{i, \mathrm{~A}}-\sum_{i=\mathrm{j}+1}^{\mathrm{m}} y_{i, \mathrm{~A}}\right)}\left[\begin{array}{c}
y_{\mathrm{O}_{2}, \mathrm{~A}} \\
y_{\mathrm{CO}_{2}, \mathrm{~A}} \\
y_{\mathrm{CO}, \mathrm{A}} \\
y_{5, \mathrm{~A}} \\
\vdots \\
y_{\mathrm{m}, \mathrm{A}}
\end{array}\right] .
$$

If one assumes $\sum_{i=\mathrm{j}+1}^{\mathrm{m}} y_{i, \mathrm{~A}} \ll 1$, Eq. (C1) can be simplified to

$$
\left[\begin{array}{c}
y_{\mathrm{O}_{2}, \mathrm{~L}} \\
y_{\mathrm{CO}_{2}, \mathrm{~L}} \\
y_{\mathrm{CO}, \mathrm{L}} \\
y_{5, \mathrm{~L}} \\
\vdots \\
y_{\mathrm{j}, \mathrm{L}}
\end{array}\right]=\frac{y_{\mathrm{N}_{2}, \mathrm{~L}}}{\left(1-y_{\mathrm{O}_{2}, \mathrm{~A}}-y_{\mathrm{CO}_{2}, \mathrm{~A}}-y_{\mathrm{CO}, \mathrm{A}}-\sum_{i=5}^{\mathrm{j}} y_{i, \mathrm{~A}}\right)}\left[\begin{array}{c}
y_{\mathrm{O}_{2}, \mathrm{~A}} \\
y_{\mathrm{CO}_{2, \mathrm{~A}}} \\
y_{\mathrm{CO}, \mathrm{A}} \\
y_{5, \mathrm{~A}} \\
\vdots \\
y_{\mathrm{j}, \mathrm{A}}
\end{array}\right] .
$$

Substituting Eq. (C2) and Eq.(52) into Eq. (3),

$$
\phi=1-\frac{n_{\mathrm{T}, \mathrm{L}} y_{\mathrm{O}_{2}, \mathrm{~L}}}{n_{\mathrm{T}, \mathrm{E}} y_{\mathrm{O}_{2}, \mathrm{E}}}=1-\frac{y_{\mathrm{N}_{2}, \mathrm{E}}}{y_{\mathrm{O}_{2}, \mathrm{E}}} \frac{y_{\mathrm{O}_{2}, \mathrm{~A}}}{\left(1-y_{\mathrm{O}_{2}, \mathrm{~A}}-y_{\mathrm{CO}_{2}, \mathrm{~A}}-y_{\mathrm{CO}, \mathrm{A}}-\sum_{i=5}^{\mathrm{j}} y_{i, \mathrm{~A}}\right)} .
$$

Substituting Eq. (71) into Eq. (C3), simplifying, and noting that $y_{\mathrm{O}_{2}, \mathrm{E}}^{*} \equiv y_{\mathrm{O}_{2}, \mathrm{E}}$ and $y_{\mathrm{N}_{2}, \mathrm{E}}^{*} \equiv y_{\mathrm{N}_{2}, \mathrm{E}}$,

$$
\phi=\frac{y_{\mathrm{O}_{2}, \mathrm{~A}}^{*}\left(1-y_{\mathrm{CO}_{2}, \mathrm{~A}}-y_{\mathrm{CO}, \mathrm{A}}-\sum_{i=5}^{\mathrm{j}} y_{i, \mathrm{~A}}\right)-y_{\mathrm{O}_{2}, \mathrm{~A}}\left(1-y_{\mathrm{CO}_{2}, \mathrm{~A}}^{*}\right)}{y_{\mathrm{O}_{2}, \mathrm{~A}}^{*}\left(1-y_{\mathrm{O}_{2}, \mathrm{~A}}-y_{\mathrm{CO}_{2}, \mathrm{~A}}-y_{\mathrm{CO}, \mathrm{A}}-\sum_{i=5}^{\mathrm{j}} y_{i, \mathrm{~A}}\right)} .
$$




\section{Journal of Research of the National Institute of Standards and Technology}

\section{References}

[1] Babrauskas V, Peacock, RD (1992) Heat release rate: The single most important variable in fire hazard. Fire Safety Journal 18:255-272. https://doi.org/10.1016/0379-7112(92)90019-9

[2] Thornton WM (1917) The relation of oxygen to the heat of combustion of organic compounds. Philosophical Magazine 33:196203. https://doi.org/10.1080/14786440208635627

[3] Babrauskas V (1984) Development of the cone calorimeter-A bench-scale heat release rate apparatus based on oxygen consumption. Fire and Materials 8(2):81-95. https://doi.org/10.1002/fam.810080206

[4] Bryant RA, Ohlemiller TJ, Johnsson EK, Hamins A, Grove BS, Guthrie WF, Maranghides A, Mulholland GW (2004) The NIST 3 Megawatt Quantitative Heat Release Rate Facility-Description and Procedures. (National Institute of Standards and Technology, Gaithersburg, MD), NIST Interagency/Internal Report (NISTIR) 7052. http://doi.org/10.6028/NIST.IR.7052

[5] Huggett C (1980) Estimation of rate of heat release by means of oxygen consumption measurements. Fire and Materials 4(2):61-65. https://doi.org/10.1002/fam.810040202

[6] Parker WJ (1982) Calculations of the Heat Release Rate by Oxygen Consumption for Various Applications. (U.S. Department of Commerce, Washington D.C.), National Bureau of Standards Interagency/Internal Report (NBSIR) 81-2427-1. http://doi.org/10.6028/NBS.IR.81-2427-1

[7] Parker WJ (1984) Calculations of the heat release rate by oxygen consumption for various applications. Journal of Fire Sciences 2(5):380-395. https://doi.org/10.1177/073490418400200505

[8] Janssens ML (1991) Measuring rate of heat release by oxygen consumption. Fire Technology 27(3):234-249. https://doi.org/10.1007/BF01038449

[9] Janssens ML, Parker WJ (1992) Oxygen consumption calorimetry. Heat Release in Fires, eds Babrauskas V, Grayson SJ (Elsevier, New York), Chapter 3, pp 31-59.

[10] Brohez S, Delvosalle C, Marlair G, Tewarson A (2000) The measurement of heat release from oxygen consumption in sooty fires. Journal of Fire Sciences 18(5):327-353. https://doi.org/10.1177/073490410001800501

[11] Chow WK, Han SS (2011) Heat release rate calculation in oxygen consumption calorimetry. Applied Thermal Engineering 31:304-310. https://doi.org/10.1016/j.applthermaleng.2010.09.010

[12] Lattimer BY, Beitel JJ (1998) Evaluation of heat release rate equations used in standard test methods. Fire and Materials 22(4):167-173. https://doi.org/10.1002/(SICI)1099-1018(1998070)22:4\%3C167::AID-FAM649\%3E3.0.CO;2-M

[13] Amundson NR (1966) Mathematical Methods in Chemical Engineering: Matrices and Their Application (Prentice-Hall, Englewood Cliffs, NJ).

[14] Himmelblau DM (1982) Basic Principles and Calculations in Chemical Engineering (Prentice-Hall, Englewood Cliffs, NJ), 4th Ed.

[15] Levenspiel O (1972) Chemical Reaction Engineering (John Wiley \& Sons, New York), 2nd Ed.

[16] Kreyszig E (1983) Advanced Engineering Mathematics (John Wiley \& Sons, New York), 5th Ed.

About the author: Jiann C. Yang is the deputy division chief in the Fire Research Division at NIST. The National Institute of Standards and Technology is an agency of the U.S. Department of Commerce. 